\title{
Modelling Guided Elastic Waves in Generally Anisotropic Media using a Spectral Collocation Method
}

\author{
F. Hernando Quintanilla ${ }^{a}$, M. J. S. Lowe ${ }^{a}$ and R. V. Craster ${ }^{b}$ \\ ${ }^{a}$ Department of Mechanical Engineering, ${ }^{b}$ Department of Mathematics, \\ Imperial College, London SW7 2AZ, UK
}

(Dated: June 8, 2015)

\begin{abstract}
Guided waves are now well established for some applications in the non-destructive evaluation of structures and offer potential for deployment in a vast array of other cases. For their development it is important to have reliable and accurate information about the modes that propagate for particular waveguide structures. Essential information that informs choices of mode transducer, operating frequencies and interpretation of signals, amongst other issues, is provided by the dispersion curves of different modes within various combinations of geometries and materials.
\end{abstract}

In this paper a spectral collocation method is successfully used to handle the more complicated and realistic waveguide problems that are required in non-destructive evaluation; many pitfalls and limitations found in root-finding routines based on the partial wave method are overcome by using this approach. The general cases presented cover anisotropic homogeneous perfectly elastic materials in flat and cylindrical geometry. Non-destructive evaluation applications include complex waveguide structures, such as single or multi-layered fibre composites, lined, bonded and buried structures. For this reason, arbitrarily multi-layered systems with both solid and fluid layers are also addressed as well as the implementation of interface models of imperfect boundary conditions between layers.

[] Accepted for publication in the Journal of the Acoustical Society of America, 137 (3), 2015.

PACS numbers: 43.35.Zc, 43.58.Ta, 43.20.Mv 


\section{INTRODUCTION}

The reliable and accurate calculation of dispersion curves plays an important role in the development of techniques for Non-Destructive Evaluation (NDE) since they allow one to rapidly identify and understand which propagating modes to utilise. The calculation of dispersion curves for waveguides of regular geometry (flat, cylindrical) is now classical and has been established by many authors. For example, with specific relevance to the present authors, the software package DISPERSE [1] and [2], developed by members of the NDE group at Imperial College London and utilised widely by both industrial and academic groups, has been a powerful tool for plotting dispersion curves in various cases such as free plates, with and without damping, fluid loaded plates, cylinders amongst others. It is also possible to select anisotropic materials although, as is typical, for guided wave modelling capabilities in general, the program is limited in its scope; for instance, when dealing with cylindrical geometries, the material must be either isotropic or transversely isotropic. Given the well-established nature of the field we begin with a short review of the area to set our work in context.

Analytical methods of solution for the simplest problems, such as that of an elastic plate in vacuum, have been known since times dating back to Lord Rayleigh. Some of these, as well as a copious bibliography, are to be found in texts such as [3], [4], [5] or [6]. Some exact solutions for isotropic media and a few standard geometries are available in textbooks or papers such as Mindlin [7] or Pao [8] and references therein. Most of the general cases for anisotropic materials in flat geometry, although widely-studied, can be found in three papers: Solie and Auld [9] studied the Christoffel equation for modes propagating at various angles in a plate of copper with cubic symmetry. Nayfeh and Chimenti [10] studied a free plate with orthorhombic symmetry and, by means of a rotation, of monoclinic symmetry as well. A more general account of anisotropic plates by the previous authors can be found in [11]. Later, Li and Thompson [12] studied in more detail materials with orthorhombic symmetry and presented various dispersion curves for a variety of orthorhombic materials at different angles of propagation; we use these later to validate our results. In NDE it is essential to treat wave propagation in cylinders and rods as applications such as pipe inspection are commonplace. The isotropic case has been extensively studied by Onoe et 
al. [13], Mindlin and Pao [14], Zemanek [15], Pao [8], or more recently Towfighi et al. [16] amongst others.

Multi-layered systems have also been extensively studied by assembling the individual solutions, via interface conditions, into matrix relationships from which a dispersion relation for the whole system emerges. Two exemplars of matrix methods for guided waves in multi-layered waveguides, taken from a rich literature over many years, are the Transfer and Global Matrix methods, described in [17]; some extensions to flat multi-layered anisotropic systems are in [18] and [19]. These approaches have all been based on the representation of the fields in layers by partial waves; they create system matrices with root-finding methods producing dispersion curves. The development of robust root-finding methods is itself a significant challenge [17]. More recently, other authors [16], [20], [21], [22] or [23] have used different methods, which will be briefly described in the paragraphs below, to handle multi-layered systems, mainly in cylindrical geometry. Partial-wave root-finding becomes cumbersome if robust solutions for general anisotropy are desired, so commonly the implementation has been restricted to isotropic, transversely isotropic, orthorhombic and monoclinic materials, [9], [10] and [12]. The upshot is that, to the best of our knowledge, there is no robust, versatile and general method available for the full anisotropic system, a situation we aim to remedy.

Three important challenges with root-finding methods emerge [17]: firstly, it is possible that root-finding routines fail to find a mode and simply skip it, jumping onto the next one, thus missing one solution. In particular, this phenomenon was observed for the general purpose root-finding routine used by the authors based on looking for minima of the dispersion relation determinant and then using a two dimensional bisecant method, a more detailed description can be found in [2]. This is normally solved by changing and adjusting the convergence parameters of the routine to make a finer search, but the authors are not aware of any successful approach to completely avoid missing roots. Secondly, we have the so-called "large $f d$ " problem; this arises from the existence in the dispersion relation of real positive and negative exponential factors which, when being too large and too small respectively, render the matrix numerically ill-conditioned. This was cured by the use of the Global Matrix Method as described in [17], and by other methods such as the 
Delta Operator method [24], the Stiffness method [25], and the Impedance method [26], although in all cases this has involved an increase in complexity of the formulation. Finally, a third issue emerges: the matrix becomes singular, yielding a spurious solution, when the wavenumber of the wave in the plate is equal to any of the bulk wavenumbers of the layers of the system. Routines can be programmed to discard such solutions straightaway, but it is troublesome to eliminate these spurious solutions when genuine modes are nearby.

An alternative, philosophically closer approach to that we advocate, is the Semi Analytical Finite Element (SAFE) method popularised by Gavric's [27] work to compute dispersion curves in a free rail. This approach allows for more geometrical flexibility than root-finding and has been successfully used in various waveguide contexts: Leaky waves travelling along arbitrary cross sectional waveguides surrounded by an infinite medium [28], torsional waves propagating along arbitrary cross sectional waveguides immersed in perfect fluid [29], trapped waves propagating along a welded joint between plates [30], guided waves in viscoelastic media with rectangular, arbitrary cross-sections [31] and axisymmetric cross-section [32].

For modelling waveguides of constant cross-section with SAFE, and limiting to flat or cylindrical geometries, one takes a one-dimensional mesh across the thickness of the system, see [32] for instance; the spectral method also uses a grid of points across the thickness but as a global interpolant rather than a local one. The SAFE method requires the use of Hamilton's Principle and finite elements to generate an eigenvalue equation, one advantage of spectral collocation vis-a-vis SAFE is the relative ease of coding and construction of the eigenvalue problem with only matrix methods required.

Probably the most important difference between these methods is the convergence rate of the spatial representation or discretisation, which impacts on the results' accuracy and speed of computation. The convergence rate of the Spectral Collocation Method (SCM) [33] is $O\left(N^{-m}\right)$ for every $m$, where $N$ is the number of grid points (or nodes) and $m$ is a constant, provided the solution is sufficiently differentiable, whereas that of SAFE and other finite element methods is only $O\left(N^{-m}\right)$ for some $m$ which depends on the order of approximation and smoothness of the function. This has serious consequences in terms 
of accuracy versus matrix size and this comes to the fore when multi-layered media are considered as eigenvalue problems remain manageable in scale. This is a consequence of the SCM using a global interpolant whilst the finite element method uses local interpolants such as piecewise polynomials; accuracy up to eleven decimal digits is commonplace using the SCM. We refer the reader to $[34,35]$ and references therein for details about the relative advantages of spectral versus finite element methods.

Finally, a Weighted Residuals Method has been used by Towfighi et al., [16], later improved by Vasudeva et al. [20], to look at annular wave propagation in anisotropic homogeneous multi-layered cylinders. The method consists of expanding the unknowns in a series of some basis functions. The residual function is calculated, and by equating to zero the suitably defined "inner product" of the residual function with the "weight or test functions" a system of algebraic functions is obtained that yield the coefficients of the expansion. This is akin to the Galerkin method for solving differential equations and can become cumbersome as one moves to more generality in anisotropy.

Given the importance of dispersion curves in this field it is unsurprising that there has been all this prior activity. However, as noted above, there is certainly scope to develop a robust, versatile and general purpose capability that allows for any kind of anisotropic homogeneous, or inhomogeneous, material in any desired orientation of the crystal axes in flat as well as cylindrical, geometry. This is particularly attractive in terms of overcoming the complexities and limitations of the partial-waves root-finding (PWRF) methods summarised above. In so doing, one would want to develop a methodology, that can be implemented to run automatically, for the complete solution without the need for any intermediate intervention from the modeller. The approach we take is the Spectral Collocation Method (SCM), a well-established approach used for the numerical solutions of differential equations. The SCM has already shown great promise when used to calculate guided wave properties for some simple elastic waveguides [22], as we will discuss shortly; our contribution here is to go much farther to develop and validate its use for general anisotropy in arbitrarily multi-layered waveguides, in both flat and cylindrical geometries.

In Section II we give a brief outline of the main characteristics and advantages of 
SCM as well as a review of the previous work done in this direction. Section III presents various cases used to validate our results and for which we can explore numerical issues of accuracy and convergence. In Section IV we cover new cases and comment upon the relevant physics and further extensions. All the physical properties used in computations are summarized in the appendix.

\section{SPECTRAL COLLOCATION METHOD (SCM)}

As this is the method we employ it is necessary to briefly explain the spectral collocation scheme and its advantages. Spectral schemes are often used for differential equations but, instead of solving a differential equation directly they use a spectral approximation for the solution that satisfies the differential equation and boundary conditions. If the problem is recast in the form of an eigenvalue problem, differentiation matrices approximate the derivative operators with spectral accuracy so there is a concise matrix eigenvalue problem that is solved for both the eigenvalues and eigenvectors. There is an extensive literature on spectral methods mainly from the point of view of solving partial differential equations where it is often the method of choice [36], [33], [34] and [35].

Although this method is well-established and has been successfully used for solving a wide range of differential equations, there has been little activity aimed at using it for dispersion curves in the field of waveguides. [22] introduced it as a potential method, and explored some simple cases, and motivated by this initial success, several papers have appeared illustrating the use of the spectral method for specific classes of waveguide problems: Karpfinger et al.,[21], have successfully used this method to handle multi-layered cylindrical systems with isotropic materials and later [37] extended this to porous elastic media and to geophysical applications involving boreholes [38]. Yu et al., [23], used the spectral method, combined with root finding routines, for multi-layered isotropic cylinders with axial propagation and weak and perfect interfaces. Finally, Zharnikov et al., [39], plot the dis-

persion curves of a waveguide of inhomogeneous anisotropic material using this methodology.

As we shall demonstrate, spectral schemes are easy to formulate and code, much more so than root finding routines, and following an entirely algebraic approach they 
are both robust and reliable. Also, if we are just interested in the lowest $M$ modes, say $M=O(10)$, they are much faster than root finding routines [22], especially for complicated problems, such as multi-layered or anisotropic materials. However, the most advantageous aspect of the SCM is that it can be deployed to model general anisotropy, and simultaneously avoid, without any special or conditional treatments, all of the challenges cited above: the SCM approach is guaranteed not to miss any of the modes, it is immune to instabilities from large values of $f d$, and it does not deliver spurious solutions at the bulk wavenumbers of the constituent materials. We will illustrate these capabilities later with suitable examples. Finally, see Boyd [35], spectral accuracy is anticipated for the first half of the eigenvalues found. A more detailed discussion on the advantages of SCM over root finding routines, with a special emphasis on elastic waveguides, is in [22].

For definiteness we consider a planar waveguide as shown in Figure 1; cylindrical cases are analogous with a change in coordinate system. Assuming a harmonic wave propagating along the $\{z\}$ axis, the equations of motion for a linear elastic anisotropic homogeneous medium are:

$$
\nabla_{i K} c_{K L} \nabla_{L j}^{s y m} u_{j}=-\rho \omega^{2} u_{i}
$$

where we use the summation convention over the indices and $c_{K L}$ is the medium's stiffness matrix in reduced index notation, [4],$u_{j}$ are the components of the displacement vector field

$$
u_{j}=U_{j}(y) e^{i(k z-\omega t)} ; \quad j=x, y, z
$$

and the differential operators are of first order in the coordinate derivatives; one finds their explicit expressions in [4]. In the following sections we will be concerned with structures in vacuum so we take traction-free boundary conditions which require the vanishing of the following three components of the stress tensor field defined below, $T_{i j}$.

Taking the faces of the plate to be located at $y= \pm h$, see Figure 1 , we summarize the boundary conditions (BCs) as:

$$
\left.T_{y y}\right|_{y= \pm h}=\left.T_{y x}\right|_{y= \pm h}=\left.T_{y z}\right|_{y= \pm h}=0 .
$$

The expression of the stress tensor field in terms of the strain tensor field reads: 


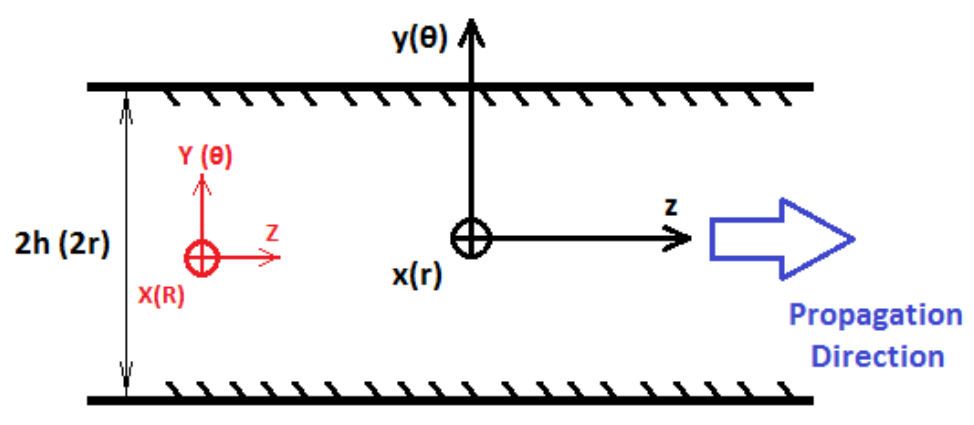

FIG. 1: Geometry and axes for flat waveguide: $\{\mathrm{X}, \mathrm{Y}, \mathrm{Z}\}$ are the crystal axes which can rotate about the fixed spatial axes $\{\mathrm{x}, \mathrm{y}, \mathrm{z}\}$ according to the choice of orientation of the material within the waveguide. In the flat case the $\{\mathrm{z}\}$ axis is the phase direction of the propagating waves (normal to the plane of the wavefront), and in the cylindrical case it is the axis of the cylinder. For the cylindrical case, $\{\mathrm{x}\}$ and $\{\mathrm{y}\}$ should be replaced by $\{\theta\}$ and $\{\mathrm{r}\}$ respectively.

$$
T_{i j}=c_{i j k l} S_{k l}
$$

Where $c_{i j k l}$ is the fourth-rank stiffness tensor, which relates to $c_{K L}$ as described in [4]. And the strain tensor field, $S_{i j}$, in terms of the displacement vector field, $u_{j}$ :

$$
S_{i j}=\frac{1}{2}\left(\frac{\partial u_{i}}{\partial x_{j}}+\frac{\partial u_{j}}{\partial x_{i}}\right) \rightarrow S_{K}=\nabla_{K j}^{s y m} u_{j}:
$$

we have a set of three equations of motion and an additional six equations for the boundary conditions. To utilise SCM we discretise and substitute the derivatives by Differentiation Matrices (DMs). Since we have a bounded interval, the appropriate choice is to use Chebyshev DMs, based on a non-uniform Chebyshev grid of $N$ points, these are $N \times N$ matrices; the generation of DMs is covered in [33], [34]. The $m$-th derivative with respect to $y$ is approximated by the corresponding $m$-th order Chebyshev DM:

$$
\frac{\partial^{(m)}}{\partial y^{(m)}} \Longrightarrow \mathbf{D}^{(m)}:=\left[D M_{C h e b}\right]_{N \times N}^{(m)}
$$

The elastic stiffness tensor does not undergo any modification; its entries multiply the matrices in the differential operators following the usual multiplication rule for tensors. From (1) we have three coupled equations, one for each component of the displacement. These are discretised with each displacement component given at the grid points and represented 
by a vector $\mathbf{U}_{j}$. Each of (1) becomes represented in matrix form as, for instance, for the $x$ component of displacement:

$$
\mathfrak{A}_{N \times N}\left(c_{K L} ; i k \mathbf{I}, \mathbf{D}^{(1)}, \mathbf{D}^{(2)}\right) \mathbf{U}_{x}+\mathfrak{B}_{N \times N} \mathbf{U}_{y}+\mathfrak{C}_{N \times N} \mathbf{U}_{z}=-\rho \omega^{2} \mathbf{U}_{x} .
$$

A similar matrix representation emerges for each of the other components of the displacement vector field. The prefactors $\mathfrak{A}_{N \times N}, \mathfrak{B}_{N \times N}, \mathfrak{C}_{N \times N}$ are $N \times N$ matrices that are formed of a linear combination of the DMs up to second degree and the identity matrix with the elastic stiffness constants, $c_{K L}$, as its coefficients.

This is a matrix system where the unknowns are the vectors $\mathbf{U}_{j}$ and the coefficients are the matrices $\mathfrak{A}_{N \times N}, \mathfrak{B}_{N \times N} \ldots$. It becomes clearer when we rearrange this system as

$$
\left(\begin{array}{ccc}
\mathfrak{A} & \mathfrak{B} & \mathfrak{C} \\
\mathfrak{D} & \mathfrak{E} & \mathfrak{F} \\
\mathfrak{G} & \mathfrak{H} & \mathfrak{I}
\end{array}\right)_{3 N \times 3 N}\left(\begin{array}{c}
\mathbf{U}_{x} \\
\mathbf{U}_{y} \\
\mathbf{U}_{z}
\end{array}\right)_{3 N \times 1}=\omega^{2}\left(\begin{array}{ccc}
-\rho \mathbf{I} & 0 & 0 \\
0 & -\rho \mathbf{I} & 0 \\
0 & 0 & -\rho \mathbf{I}
\end{array}\right)\left(\begin{array}{c}
\mathbf{U}_{x} \\
\mathbf{U}_{y} \\
\mathbf{U}_{z}
\end{array}\right)
$$

Or more concisely:

$$
\mathfrak{L}(k) \mathbf{U}=\omega^{2} \mathfrak{M} \mathbf{U}
$$

Where $\mathbf{U}$ is the vector of vectors: $\mathbf{U}=\left[\mathbf{U}_{x}, \mathbf{U}_{y}, \mathbf{U}_{z}\right]^{T}$. Before proceeding we must include the boundary conditions; the six equations (3) are discretised and rearranged, as in [22] or [33], so

$$
\mathfrak{S}(k):=\left(\begin{array}{ccc}
\mathfrak{T}_{A} & \mathfrak{T}_{B} & \mathfrak{T}_{C} \\
\mathfrak{T}_{D} & \mathfrak{T}_{E} & \mathfrak{T}_{F} \\
\mathfrak{T}_{G} & \mathfrak{T}_{H} & \mathfrak{T}_{I}
\end{array}\right)\left(\begin{array}{c}
\mathbf{U}_{x} \\
\mathbf{U}_{y} \\
\mathbf{U}_{z}
\end{array}\right)=\left(\begin{array}{l}
0 \\
0 \\
0
\end{array}\right)
$$

Then, we return to $(8)$ and replace the $1, N,(N+1), 2 N,(2 N+1)$ and $3 N$ rows of the $\mathfrak{L}$ matrix with those of the $\mathfrak{S}$ matrix of (10). These rows correspond, for each component of the displacement vector field, to the grid points $y= \pm h$, that is, rows 1 and $N$ go with $\mathbf{U}_{x}$ evaluated at $y=h$ and $y=-h$ respectively, rows $N+1$ and $2 N$ go with $\mathbf{U}_{y}$ evaluated at $y=h$ and $y=-h$ and so on. Similarly, we replace the same rows of the matrix $\mathfrak{M}$ on the right hand side with rows filled with zeroes. The resultant system then has both the governing equation and boundary conditions incorporated in a consistent manner and this is then a standard generalised eigenvalue problem ideally suited for coding; we used MATLAB (version R2012b) and the eigenvalue routine eig which, given a value of $k$, provides the 
angular frequencies, $\omega$, of the first $N$ modes. As an added bonus the eigenvectors $\mathbf{U}$ contain the mode shapes.

Multiple layers follow this methodology and we assemble as many systems, using (8), as the number of layers in the system, and then compose the boundary conditions. The boundary conditions are still given by equations (3), but for layers we also impose continuity at the interfaces for the displacement fields as well as for the three components of the stress tensor field.

As a specific example consider a system of $n$ layers of triclinic material, the equation analogous to (9) is:

$$
\left(\begin{array}{cccc}
\mathfrak{L}_{1} & \mathcal{0} & \ldots & 0 \\
\mathfrak{o} & \mathfrak{L}_{2} & \ldots & 0 \\
\vdots & \vdots & \ddots & \vdots \\
\mathfrak{0} & \mathcal{0} & \ldots & \mathfrak{L}_{n}
\end{array}\right)_{3 n N \times 3 n N}\left(\begin{array}{c}
\mathbf{U}_{1} \\
\mathbf{U}_{2} \\
\vdots \\
\mathbf{U}_{n}
\end{array}\right)_{3 n N \times 1}=\omega^{2}\left(\begin{array}{cccc}
\mathfrak{M}_{1} & \mathcal{0} & \ldots & 0 \\
\mathfrak{o} & \mathfrak{M}_{2} & \ldots & 0 \\
\vdots & \vdots & \ddots & \vdots \\
\mathcal{0} & \mathcal{0} & \ldots & \mathfrak{M}_{n}
\end{array}\right)_{3 n N \times 3 n N}\left(\begin{array}{c}
\mathbf{U}_{1} \\
\mathbf{U}_{2} \\
\vdots \\
\mathbf{U}_{n}
\end{array}\right)_{3 n N \times 1}
$$

Each of the labelled matrices: $\mathfrak{L}_{i}, \mathfrak{M}_{i}$ and $\mathbf{U}_{i}$ (where $i=1 . . N$ indicates the block matrices, vectors for the displacements etc in each layer $i$ ) has the single layer form shown in equation (9).

Having arranged the boundary conditions for a single layer in matrix form in equations (10), for the external boundary conditions we proceed in a similar fashion to that outlined for a single layer. For the upper layer we have its corresponding boundary condition matrix $\mathfrak{S}_{1}$ whose $1,(N+1)$ and $(2 N+1)$ rows give the three components of the stress tensor field at the upper external boundary (3). These three rows replace the first three rows $1, N$ and $(N+1)$ of the multilayer system matrix (11). One follows an analogous procedure for the bottom external boundary: take the $N, 2 N$ and $3 N$ rows of the bottom layer boundary condition matrix $\mathfrak{S}_{n}$ and replace the $((3 n-1) N),((3 n-1) N+1)$ and $3 n N$ of the multilayer system matrix (11) with them.

We also impose continuity conditions across the internal interfaces. For an interface, say first interface between layers 1 and 2, the continuity conditions for the stress tensor 
field are:

$$
\begin{aligned}
& \left(T_{y y}\right)_{(1)}-\left.\left(T_{y y}\right)_{(2)}\right|_{y=1^{s t}}=0 \\
& \left(T_{y x}\right)_{(1)}-\left.\left(T_{y x}\right)_{(2)}\right|_{y=1^{s t}}=0 \\
& \left(T_{y z}\right)_{(1)}-\left.\left(T_{y z}\right)_{(2)}\right|_{y=1^{s t}}=0
\end{aligned}
$$

Where $y=1^{\text {st }}$ means evaluation at the first interface. The continuity conditions in a perfect interface for the displacement fields are:

$$
\begin{aligned}
& \left(u_{x}\right)_{(1)}-\left.\left(u_{x}\right)_{(2)}\right|_{y=1^{s t}}=0 \\
& \left(u_{y}\right)_{(1)}-\left.\left(u_{y}\right)_{(2)}\right|_{y=1^{s t}}=0 \\
& \left(u_{z}\right)_{(1)}-\left.\left(u_{z}\right)_{(2)}\right|_{y=1^{s t}}=0
\end{aligned}
$$

This gives a total of six equations for each interface. Therefore for a system of $n$ layers, we have $n-1$ interfaces and $6 n-6$ interface equations, plus 6 boundary condition equations make a total of $6 n$ equations that must replace the appropriate rows in the system matrix (11).

We now specialise to a concrete example including interface conditions: Lamb modes in a flat plate with the axes configuration of Figure 1. Lamb modes decouple from $\mathrm{SH}$ modes so, for this example, there are only two displacement fields, $u_{y}$ and $u_{z}$, and the associated stress tensor field components $T_{y y}$ and $T_{y z}$. The matrix incorporating the interface and boundary lines is shown in Figure 2 which is easily extended to more general cases.

The construction of the matrix is absolutely key to the method and so we describe it in detail. In Figure 2, the superscripts a,b,c and $d$ make reference to the different elements of the matrix expression for the given $j$-th layer $\mathfrak{L}_{j}$ which forms the blocks of the whole system matrix in (11). Each of these layer matrices is a $2 N$ by $2 N(3 N$ by $3 N$ in the general case) non-diagonal matrix, and is, in turn, composed of four different sub-blocks. The a and b sub-blocks incorporate the equation of motion for the $U_{y}$ component: the a subblock acts upon the $U_{y}$, whereas the b sub-block acts upon the $U_{z}$. The c and d sub-blocks incorporate the equation of motion for the $U_{z}$ component: the c sub-block acts upon the $U_{y}$, whereas the d sub-block acts upon the $U_{z}$. The placing of the boundary conditions in 
matrix expressions in general using the Spectral Method is also explained and discussed in detail in [33]. To make this layered structure of the Matrix more apparent, we have enclosed in boxes of different colours (green for the first layer (top), brown for the second and purple for the $n$-th layer (bottom)) the blocks of the matrix corresponding to different layers.

In Figure 2, the first two rows of the system matrix are replaced by the 1-st rows of the tensor components $T_{y y}$ and $T_{y z}$ evaluated at the top of the first layer (the top boundary of the system), labelled with a 1. An analogous substitution is made in the last two rows for the $n-t h$ layer. But now we take the bottom $N$-th row of the matrices since the stress tensor field components are being evaluated at the bottom of the layer (the bottom boundary of the system). The boundary conditions are thus accounted for, and in Figure 2 these are framed in red boxes.

The first set of interface conditions corresponding to the first interface of the system linking layers 1 and 2 is framed inside orange boxes in Figure 2. These make a total of four equations: two for the displacement vector field and two for the stress tensor field. Let us begin with the continuity of $T_{y y}$ at the interface: we will replace the $(N+1)$-th row of the system with this continuity condition as shown in Figure 2. Note that this condition comprises two parts: the first refers to the bottom of layer 1 , so we take the bottom row, $N$-th, of the matrix expression for the stress tensor field $T_{y y}$ in layer 1. The second refers to the top of layer 2 so we take the top row, 1-st, of the matrix expression of $T_{y y}$ in layer 2. One should not forget the relative sign between them. We make an analogous substitution for $T_{y z}$ and replace the $(2 N)$-th row of the system.

The second set of interface conditions are the continuity conditions for the displacement vector field. The system matrix is acting upon the components of the vector field within each layer (recall the column vector to the right of the matrix in (11)), so wherever we see a $\mathfrak{U}_{j^{(y)}}(N)$ it must be understood that it is the corresponding row of the identity matrix we are dealing with, in this case it would be the bottom row. For $U_{y}$ there are again two parts: the first refers to the bottom of layer 1 , so we take the bottom $N$-th row of the identity matrix which means we are taking $U_{y}$ evaluated at the bottom of the layer 1 . The second refers to the top of layer 2 so we must take the first row of the identity matrix 


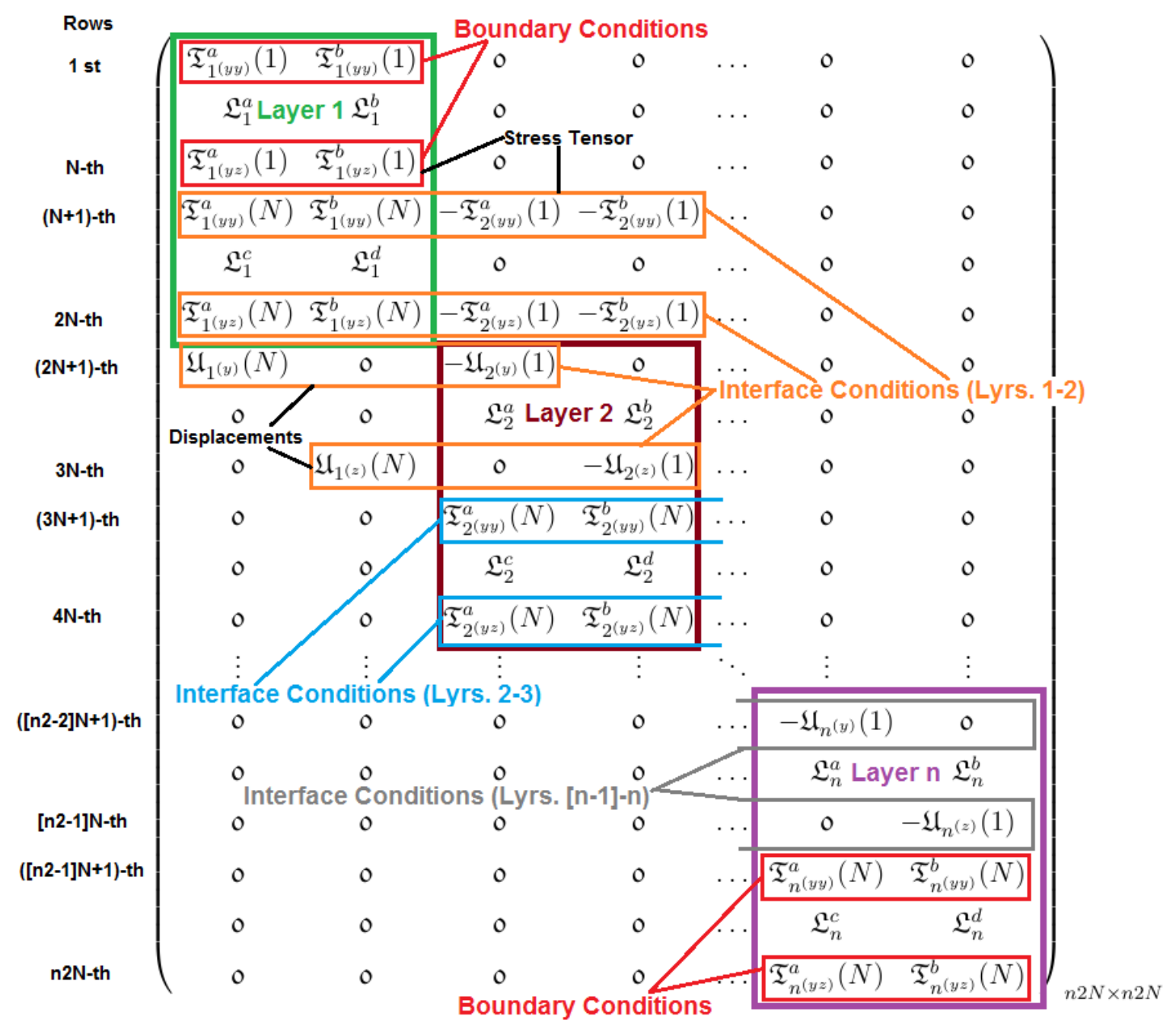

FIG. 2: Collocation scheme for the $n$-layer system matrix. The scheme shown here corresponds to a case where only two displacement fields enter the equations, such as Lamb modes. Only the first, second, and last layers are shown in detail. Analogous blocks model the rest of the layers.

which means we are taking $U_{y}$ evaluated at the top of layer 2, with this we have finished the substitutions on the $(2 N+1)$-th row of the system. A similar procedure will account for the $U_{z}$ component. One then deals with the remaining interfaces in an analogous fashion. In Figure 2 are some indications for the second and $(n-1)$-th interfaces.

A common example of interest is that of an inviscid fluid layer in between solid layers, for example the important situation of a fluid filled pipe falls into this category. Whether in flat or cylindrical geometry, this requires a modification of the scheme described 
so far as the fluid does not support shear: we retain the displacement fields for the adjacent solid layers, thus allowing us to handle any kinds of material and orientation of the crystal within the solid layers, but we use a longitudinal (compressional) potential for the fluid layer. Perfect fluids can be modelled as isotropic solids with the shear stiffness constant $\mu$ equal to zero, see [2] and references therein. Failure to do so leads to an error in the fluid-solid boundary conditions giving spurious modes and the distortion of the correct modes in the vicinity of the bulk wavenumber of the fluid.

Cylindrical cases are completely analogous with cylindrical coordinates replacing Cartesians. We study all the wave families present in cylindrical geometry: Torsional, Longitudinal and Flexural modes. The ansatz used in the early literature [3] for Flexural modes in terms of sines and cosines is not appropriate for more general anisotropic materials such as Triclinic since these terms cannot be taken out as common factors. Therefore, to handle the full range of materials, we use the general form

$$
u_{j}=U_{j}(r) e^{i(k z+n \theta-\omega t)} ; \quad j=r, \theta, z
$$

Finally, often a spring interface condition is used when interfaces with finite stiffness need to be modelled, for example when a sufficiently thin adhesive bond can be approximated by a compliant boundary or simply when an imperfect contact condition must be accounted for (sliding boundaries or partially-contacting boundaries for example). These require a slight modification of the internal interface conditions based on the spring model developed by Rokhlin and Wang [40], we refer the reader to their paper for details of the derivation. In their model it is only the continuity conditions for the displacement fields that are modified, so, instead of having equations (13) we have (recalling that the upper layer is labelled with (1) and the lower with (2)):

$$
\begin{aligned}
& {\left.\left[\frac{\left(T_{y y}\right)_{(1)}}{K_{n}}-\left(\left(u_{y}\right)_{(1)}-\left(u_{y}\right)_{(2)}\right)\right]\right|_{y=1^{s t}}=0} \\
& {\left.\left[\frac{\left(T_{y x}\right)_{(1)}}{K_{t}}-\left(\left(u_{x}\right)_{(1)}-\left(u_{x}\right)_{(2)}\right)\right]\right|_{y=1^{s t}}=0} \\
& {\left.\left[\frac{\left(T_{y z}\right)_{(1)}}{K_{t}}-\left(\left(u_{z}\right)_{(1)}-\left(u_{z}\right)_{(2)}\right)\right]\right|_{y=1^{s t}}=0}
\end{aligned}
$$

where $K_{n}$ and $K_{t}$ are the normal and transverse stiffness coefficients, which according to the definition in [40], have dimensions of $N / m^{3}$. It is straightforward to account for this 
modification; we only need to modify the corresponding rows of the system matrix.

Spring models are useful in practise since, when this representation is valid, it replaces a very thin layer by spring interface conditions thereby reducing the size of the matrix system. For example, two steel plates bonded by a thin EPOXY adhesive layer can be represented as a two layer system with a spring model instead of having a three layer system with perfect internal interface conditions; such an approximation is only valid for very thin interface layers.

\section{COMPARING WITH EXISTING RESULTS}

We validate the scheme by choosing exemplar cases of dispersion curves from the literature that illustrate important points, we use a root-finding algorithm to provide comparisons to compare with SCM. We address flat plates first, then torsional and longitudinal modes in cylinders, then the more complex case of flexural modes in cylinders. In section IV we then move on to calculate dispersion curves for cases not studied before.

The axis configuration for the cases with plane geometry is as follows: we take the axes $x$ and $z$ to lie within the plane of the plate, see Figure 1, and the axis $y$ along the thickness of the plate; wherever this is not the case we will state it explicitly. The propagation direction will invariably be along the $z$ axis unless otherwise stated. For different configurations we rotate the crystal axes whilst preserving the propagation direction.

\section{A. Free Anisotropic and Homogeneous Plate}

We begin by finding the modes for the single-layer flat plate in vacuum: In principal orientation configuration, Lamb (in-plane) modes and SH (out-of-plane) modes are found. We also present dispersion curves for configurations with arbitrary orientation of the crystal axes where modes are coupled. Following (2), we assume the form of the displacement field is the following:

$$
u_{j}=U_{j}(y) e^{i(k z-\omega t)} ; \quad j=x, y, z
$$


For an isotropic plate, SH modes are not coupled to the Lamb modes. This splitting of modes also occurs in certain cases for anisotropic materials as shown in Table I. This table is constructed using the software Maple (version 17), for the axes configuration shown in Figure 1. The transformations refer to the crystal axes rotating about the spatial $\{y\}$ axis.

Similar tables can be built for the other two possible configurations $(\{x\}$ or $\{z\}$ axis perpendicular to the plane of the plate) with analogous results. For more general rotations, e.g. two consecutive rotations different from 90 degrees about different axes, $\mathrm{SH}$ and Lamb modes are then coupled and each of these cases must be studied separately.

\begin{tabular}{|l|l|}
\hline Crystal & Transformation \\
\hline Isotropic & Lamb and SH modes decouple for any angle. \\
\hline Cubic & Lamb and SH modes decouple for 0 and 90 degrees. \\
\hline Hexagonal & Lamb and SH modes decouple for 0 and 90 degrees. \\
\hline Tetragonal 6 & Lamb and SH modes decouple for 0 and 90 degrees. \\
\hline Tetragonal 7 & Lamb and SH modes decouple only for 90 degrees. \\
\hline Trigonal 6 & Lamb and SH modes decouple only for 0 degrees. \\
\hline Trigonal 7 & No modes decouple unless one chooses the axes orien- \\
\hline tation in which Trigonal 7 takes the form of Trigonal \\
\hline Orthogonal 9 & Lamb and SH modes decouple for 0 and 90 degrees. \\
\hline Monoclinic 13 & No modes decouple. \\
\hline Triclinic 21 & No modes decouple. \\
\hline
\end{tabular}

TABLE I: Conditions for decoupling mode families in the different classes of anisotropic crystals.

We first focus on materials with Orthorhombic symmetry which have nine different constants in the stiffness matrix: $c_{11}, c_{12}, c_{13}, c_{22}, c_{23}, c_{33}, c_{44}, c_{55}$ and $c_{66}$ (in reduced index notation, see [4] for details). These materials have been extensively studied by Li and Thompson [12] and Nayfeh and Chimenti [10] and provide a challenging test for our methodology. In addition, we have compared our results to those given by PWRF methods always finding excellent agreement. 
Figure 3 compares the results obtained by the SCM with those obtained by PWRF for the case of Lamb modes in a single plate in vacuum. The plate is Orthorhombic (more details may be found in the appendix for this and the forthcoming figures) and the crystal axes are rotated such that the crystal $\{Z\}$ axis aligns with the $\{z\}$ of the plate (the propagation direction), while the crystal $\{\mathrm{X}\}$ axis aligns with the $\{\mathrm{y}\}$ axis of the plate (normal to the plate).

As can be seen in Figure 3 all the modes were found, and the SCM's solutions (red circles) lie very close to corresponding locations on the lines plotted by the general purpose PWRF routines used by the authors. As a point of note, the PWRF routine did not find the fundamental extensional mode (the mode crossing the phase velocity axis of the plot at $1.5 \mathrm{~m} / \mathrm{ms}$ ). Although it is easily found by performing a finer search using PWRF the SCM did not miss it. This illustrates the important capability of the SCM to find all modes automatically without missing any.

The configuration of Figure 3, with the crystal axes aligned with the spatial axes, implies, according to Table I, that the Lamb and SH modes decouple; for clarity and brevity we only show the Lamb modes which is the more complicated case. The SH modes are easy to compute, and compare, and equally satisfactory results are found.

Figure 4 shows the result obtained with the SCM for a reproduction of the Figure 14 from Li and Thompson's paper [12] displaying modes in an Orthorhombic plate in vacuum. The crystal axes configuration is as follows: the $Z$ axis is perpendicular to the plane of the plate and with the propagation direction at 30 degrees with respect to the $X$ axis. As a result of this rotation the stiffness matrix which enters the equations of motion has Monoclinic symmetry, so we can regard this case either as a rotated Orthorhombic example, or as an example of a Monoclinic material. This case is of particular importance because it constitutes a necessary step before studying the most general problem of Triclinic media which must contain Monoclinic materials as one of the limiting cases. The dispersion curves obtained by the SCM are identical to those shown in the paper. Since we can regard this configuration as that of a Monoclinic material, its properties apply and according to 


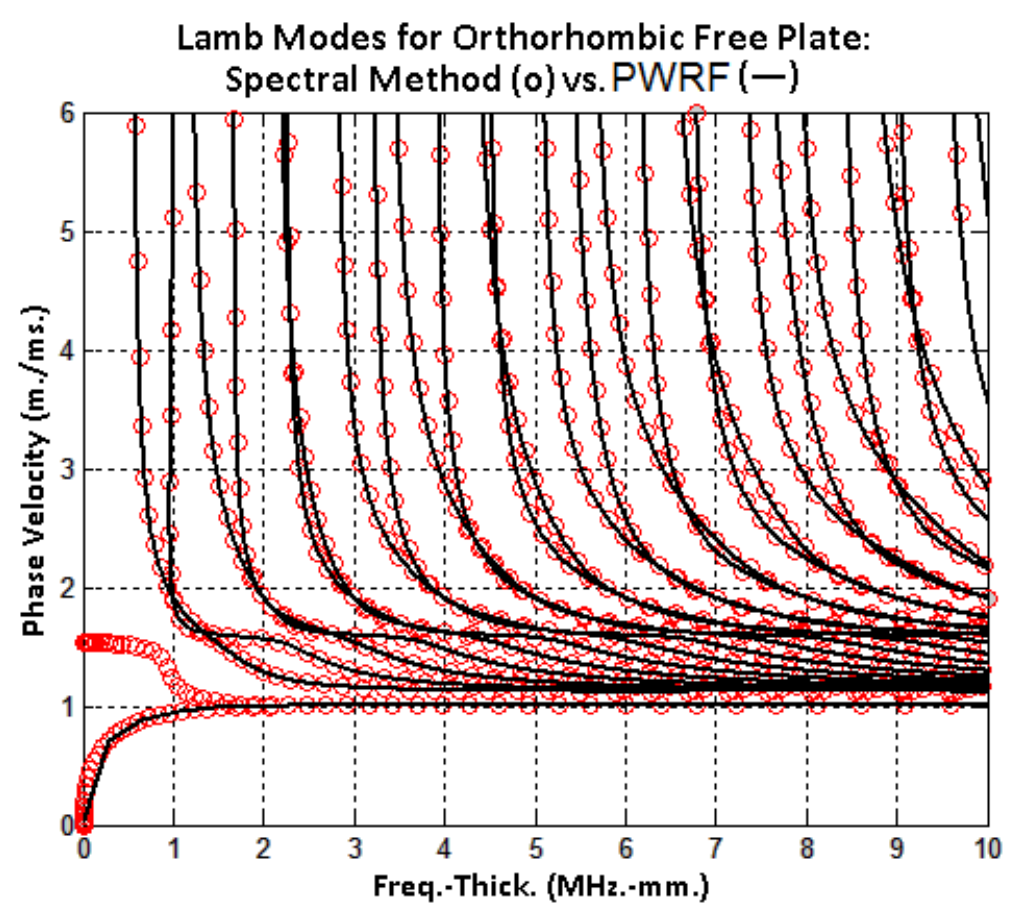

FIG. 3: Free Plate Orthorhombic Lamb Modes: SCM (circles) vs. PWRF (solid lines). Geometry and spatial axes configuration as in Figure 1. The crystal axes have been rotated to match the configuration followed by the PWRF model: propagation along the $\{z\}$ axis and the $\{x\}$ axis perpendicular to the plane of the plate. The SH family of modes in this case are uncoupled from the Lamb modes, and have not been included in the plot, although they may be obtained with similar accuracy.

Table I, Lamb and SH modes are coupled. Further rotating the crystal about an axis different from the $\{Z\}$ axis will result in a stiffness matrix of a Triclinic Material. We can therefore generalize our methodology to Triclinic materials, which is a generality that has not been treatable with other methods.

Figure 5 shows the coupled modes computed by the SCM in a flat free Triclinic plate and the axes orientation is as described in Figure 1: propagation along the $\{\mathrm{Z}\}$ axis and $\{\mathrm{Y}\}$ axis perpendicular to the plane of the plate, in this case the crystal axes and spatial axes are aligned. The physical parameters used are given in the appendix.

This is an important, and to the best of our knowledge, new result: It is the most 


\section{Modes for Free Orthorhombic Plate with the Spectral Method}

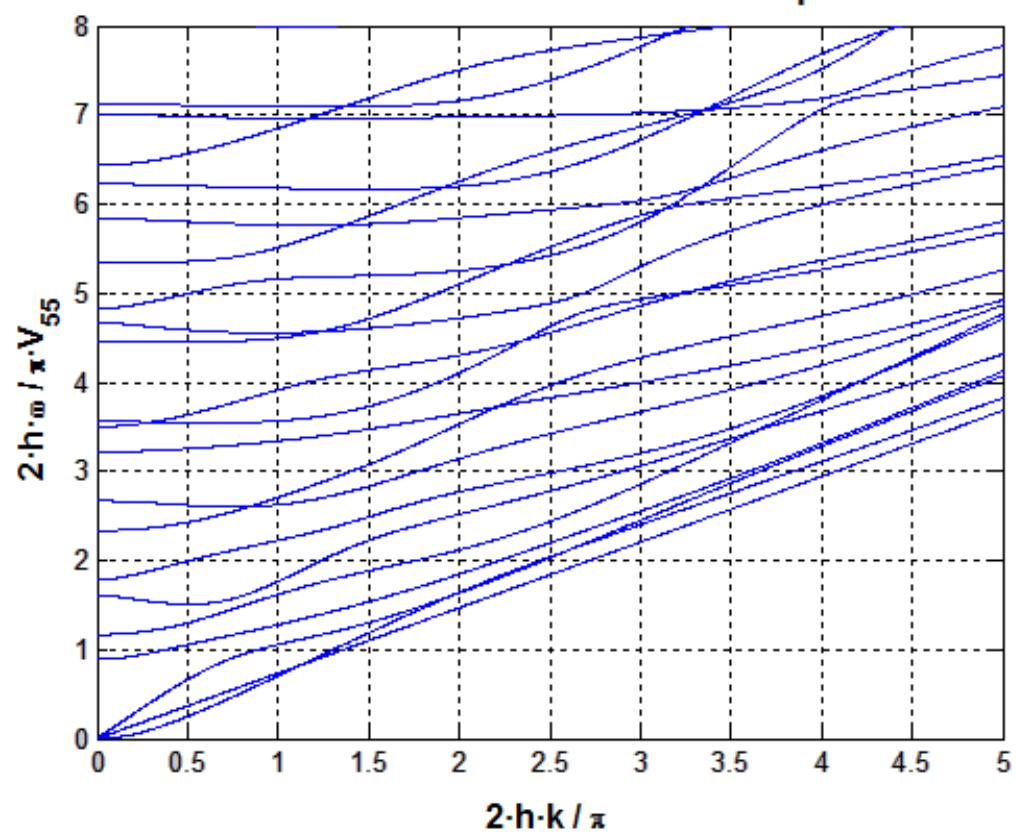

FIG. 4: Reproduction with the SCM of the results of Figure 14 in the paper by Li and Thompson [12]. This is for an orthorhombic free plate, the $\{\mathrm{Z}\}$ axis of the crystal is perpendicular to the plate and the propagation direction is at 30 degrees with respect to the $\{\mathrm{X}\}$ axis. More details about the parameters may be found in the appendix. In the graph, the $\{y\}$ axis is a normalized adimensional frequency and the $\{\mathrm{x}\}$ axis is a normalized adimensional wavenumber.

general material symmetry we can have with 21 different constants in the stiffness matrix and therefore, we can also make use of the Triclinic algorithm to solve problems of simpler symmetry (orthorhombic for instance) but with completely arbitrary orientations.

\section{B. Free Anisotropic and Homogeneous Cylinder: Torsional and Longitudinal} Modes

In cylindrical geometry the axes configuration is the conventional one: taking the axis of the cylinder along the $\{z\}$ axis. The form of the solution is as follows:

$$
u_{j}=U_{j}(r) e^{i(k z-\omega t)} ; \quad j=r, \theta, z
$$

We will focus first on the Longitudinal modes, these are analogous to the Lamb modes in flat geometry. When possible, PWRF routines will be used to test the results given by 


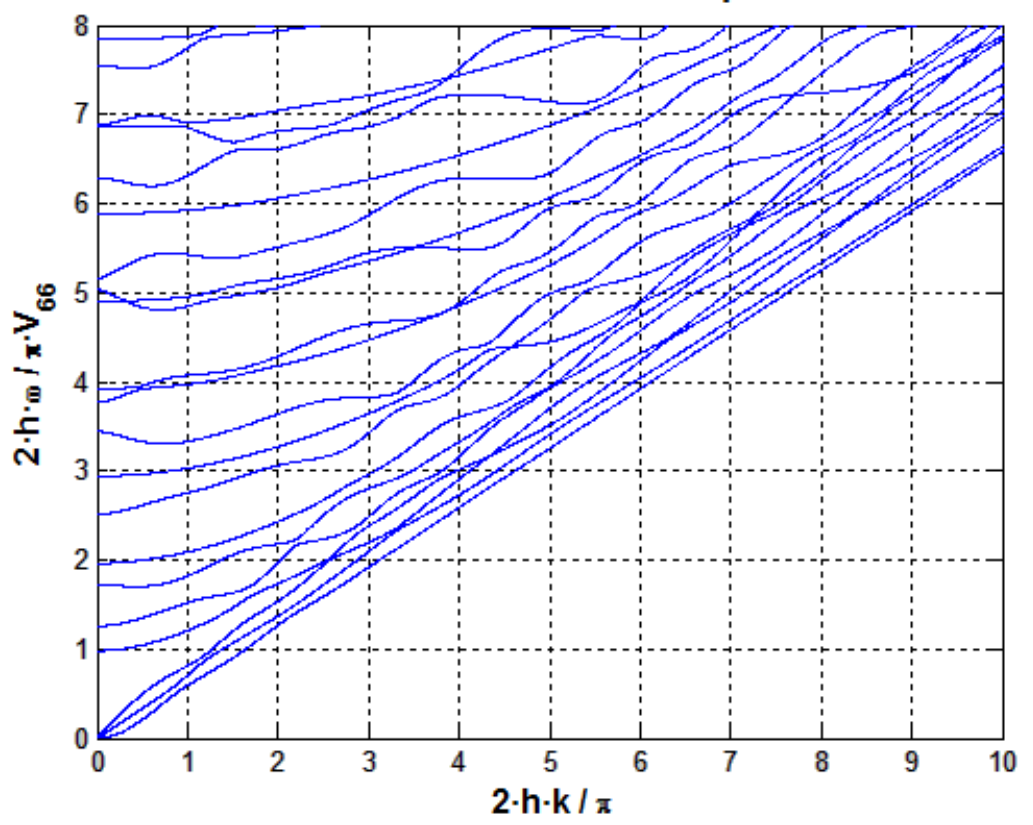

FIG. 5: Coupled modes in Triclinic free Plate obtained by the SCM with the spatial axes' orientation shown in Figure 1. The crystal axes coincide with the spatial axes. The graph displays again normalized adimensional frequency and wavenumber in the $\{y\}$ and $\{x\}$ axis respectively.

the SCM, however, their capabilities are more limited in cylindrical geometry than in flat geometry. When PWRF cannot provide us with a reliable solution and there is no available data in the literature, we can use the dispersion curves of flat plates to investigate our results' accuracy in cylindrical geometry. This is possible because we may take the thin plate limit by making $h / r \ll 1$, where $h$ stands for thickness and $r$ for internal radius of the cylinder, and expect to obtain the same dispersion curves of a plate with similar physical properties. In this limit, the Torsional and Longitudinal modes look like the SH and Lamb modes of a flat plate respectively. Note that, this limit is valid as long as the wavelength of the guided wave remains small compared to the radius of the cylinder and therefore the effects of the curvature (inverse of the radius) can be neglected. An exhaustive study of this and other limiting cases and its ranges of validity lies out of the scope of this paper but the interested reader can find a full account of them and the latest developments regarding thin shell approximations and related topics are addressed in detail in recent books by Kaplunov et al. [42] and more recently by Wang et al. [43]. However, for our validating 
purposes, the conventional thin plate limit is sufficient to test the good performance of the codes as will be seen in the next sections.

The solutions for the isotropic cylinder were easily solved by the SCM and upon comparison with available data from the literature and PWRF perfect agreement was found between both sets of dispersion curves; for brevity these are not shown here and we move on to the results for more complicated problems that cannot be solved by PWRF. To demonstrate the generality of our approach Figure 6 displays dispersion curves of a Triclinic cylinder in the Thin Plate Limit (circles) and those of the corresponding flat Triclinic plate (solid lines). This example is, to the best of our knowledge, also new and also provides a consistency check for the flat plate since both solutions show excellent agreement as expected. The axes configuration is as follows: propagation along the principal crystal axis $\{Z\}$; crystal axes are aligned with spatial axes $\{r\},\{\theta\}$ and $\{z\}$.

\section{Free Anisotropic and Homogeneous Cylinder: Flexural Modes}

Continuing the study of cylindrical geometries we move on to Flexural modes. The solutions for isotropic materials were successfully reproduced by the SCM so we focus on materials with triclinic symmetry due to their importance. Our SCM results displayed the typical behaviour of flexural modes: the higher the value of the harmonic order $n$ is, the higher the cut-off frequency of the first mode. The curves were smooth and the pattern was similar to the flexural modes for isotropic materials encountered in the literature [14] and $[15]$.

In order to verify our methodology for the Flexural modes we used the previously studied cases both in flat and cylindrical geometry. This verification proceeds in two steps. Firstly, in the Thin Plate Limit we confirm that the Flexural modes, regardless of the harmonic order $n$ chosen, agree with those of a plate of the same properties. This independence of the dispersion curves with respect to the harmonic order $n$ in the Thin Plate Limit reflects the fact that in a flat plate there is no angular dependence whatsoever and, although our equations for the flexural modes depend on the harmonic order parameter $n$, upon taking the Thin Plate Limit our results should show complete independence of 


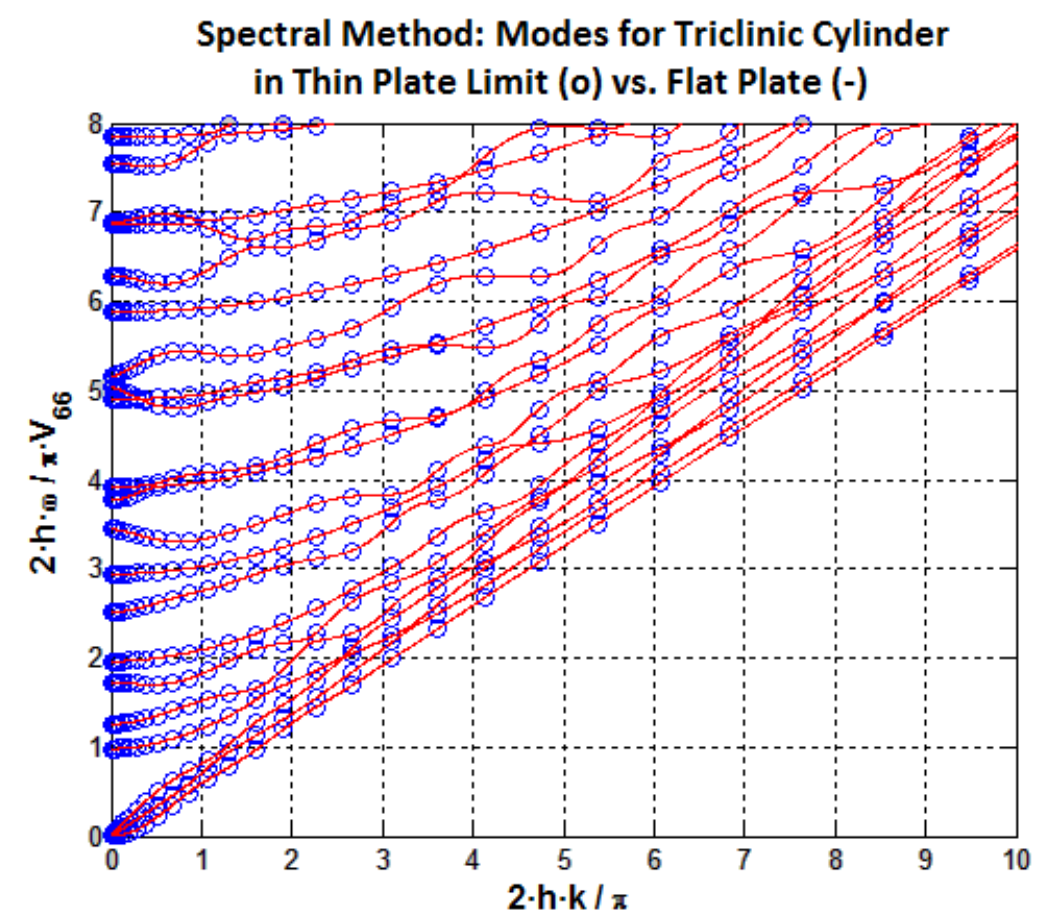

FIG. 6: Coupled modes in Triclinic Cylinder in Thin Plate Limit (circles) vs. Triclinic Plate (solid lines). The spatial axes' orientation is shown in Figure 1. The crystal axes coincide with the spatial axes. The graph displays normalized adimensional frequency and wavenumber in the $\{\mathrm{y}\}$ and $\{\mathrm{x}\}$ axis respectively. Both solutions were computed by the SCM.

it and this is what we encountered. Secondly, we corroborate that the family of flexural modes $n=0$ agrees with the Torsional and Longitudinal modes of an identical cylinder without restricting ourselves to the Thin Plate Limit.

Figure 7 shows the dispersion curves for the family $n=3$ of Flexural modes of a free Triclinic cylinder in the Thin Plate Limit (circles) compared to the results in a free plate of similar properties and equal thickness, $5 \mathrm{~mm}$ (solid lines). All the details may be found in the appendix at the end of this article. The axes' configuration is with wave propagation along the principal axis $\{Z\}$. Crystal axes are aligned with spatial axes $\{\mathrm{r}\}$, $\{\theta\}$ and $\{z\}$. Note that the axes in the plate must match those in the cylinder for the comparison to be meaningful. The plate configuration is the same as that used for Figure 5. The results for the $n=3$ Flexural modes in the cylinder agree perfectly with the dispersion curves of a flat plate of similar characteristics. 


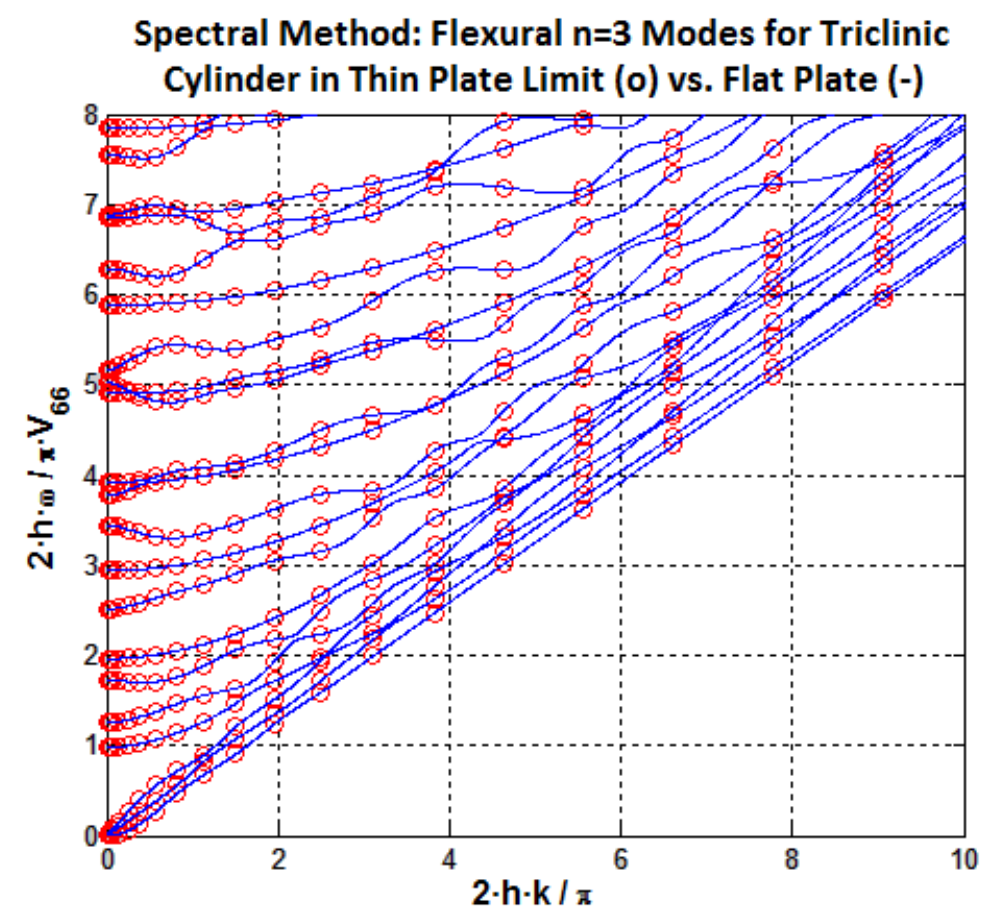

FIG. 7: Free Triclinic Cylinder: Thin Plate Limit Flexural Modes $n=3$ (circles) vs. Plate Coupled Lamb and SH Modes (solid lines). The spatial axes' orientation is shown in Figure 1. The crystal axes coincide with the spatial axes. The graph displays normalized adimensional frequency and wavenumber in the $\{\mathrm{y}\}$ and $\{\mathrm{x}\}$ axis respectively. Both solutions were computed by the SCM.

We continue verifying that the $n=0$ family of Flexural modes agrees with the Longitudinal and Torsional modes of a similar cylinder. These modes are now coupled since we are dealing with a Triclinic material. The results are plotted in figure 8. Note that the cylinder is no longer in the Thin Plate Limit but has internal radius $r=100 \mathrm{~mm}$ and wall thickness $h=100 \mathrm{~mm}$. The axes configuration is again with wave propagation along the principal axis $\{\mathrm{Z}\}$ and crystal axes aligned with spatial axes $\{r\},\{\theta\}$ and $\{z\}$. Agreement between both solutions is again excellent. Having thus verified the code we can be confident that the results for different configurations and materials will be correct.

Notably we can treat solid rods using this methodology by choosing a very small inner radius $\backsim 10^{-12}$, however, for the sake of brevity, we do not present the results of their verifications here. 


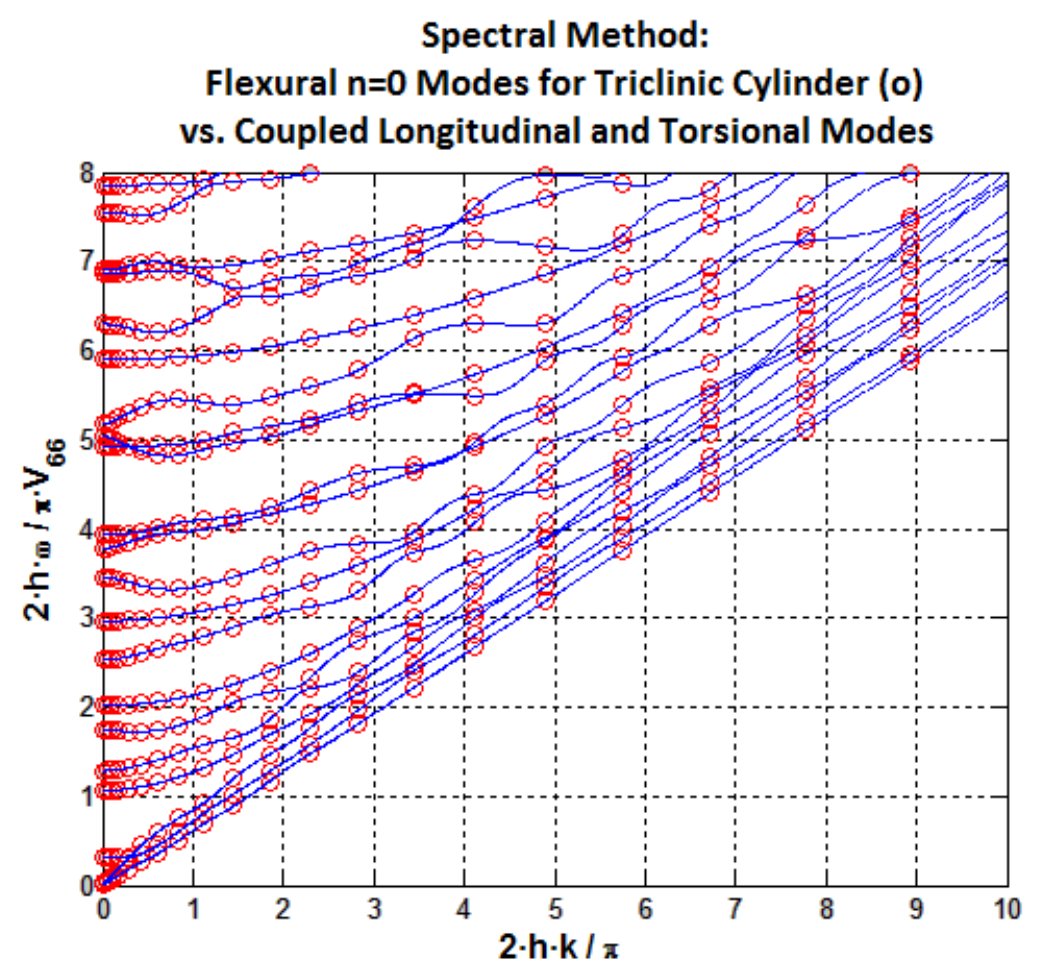

FIG. 8: Free Triclinic Cylinder: Flexural Modes $n=0$ (circles) vs. Coupled Longitudinal and Torsional Modes (solid lines). The spatial axes' orientation is shown in Figure 1. The crystal axes coincide with the spatial axes. The graph displays normalized adimensional frequency and wavenumber in the $\{y\}$ and $\{\mathrm{x}\}$ axis respectively. Both solutions were computed by the SCM.

\section{Convergence of Results}

An important feature of SCM is that the first half of the $N$ eigenvalues found are highly accurate, see for example [35] and [22]. In both references it is shown that roughly the first $N / 2$ of the eigenmodes found should be regarded as reliable and accurate, the rest may be discarded.

In Table II we assess the accuracy of the SCM for the case previously shown in Figure 3 by comparing the numerical values from the first 5 eigenmodes with those obtained by PWRF. The SCM was run with $N=70$.

Due to the large amount of results presented it is not possible to give an account of 


\begin{tabular}{|c|c|c|c|c|}
\hline Mode & $\begin{array}{r}\text { Freq.-Thick. } \\
(\mathrm{MHz}-\mathrm{mm})\end{array}$ & $\begin{array}{c}\text { PWRF } \\
V_{p h}(\mathrm{~m} / \mathrm{ms})\end{array}$ & $\begin{array}{c}\text { S. M. }(N=70) \\
V_{p h}(\mathrm{~m} / \mathrm{ms})\end{array}$ & $\begin{array}{c}\text { Relative Error (R.E) } \\
10^{-3} \%\end{array}$ \\
\hline $1^{\text {st }}$ & 0.61227 & 0.87418 & 0.87419 & 1.14391 \\
\hline $2^{\text {nd }}$ & 0.92790 & 1.32481 & 1.32483 & 1.50963 \\
\hline $3^{\text {rd }}$ & 1.16137 & 1.65818 & 1.65818 & 0 \\
\hline $4^{\text {th }}$ & 1.20336 & 1.71814 & 1.71813 & 0.58203 \\
\hline $5^{t h}$ & 1.67359 & 2.38950 & 2.38951 & 0.41849 \\
\hline
\end{tabular}

TABLE II: Relative error between the results given by the SCM and PWRF for the case previously shown in Figure 3. The values correspond to the first 5 eigenvalues.

their accuracy for each one, but whenever they were compared to PWRF very good agreement was found for the first $N / 2$ eigenvalues and Table II is typical. Nevertheless, we discuss some features of accuracy and convergence in more detail. We distinguish between two cases: first, problems, such as $\mathrm{SH}$ waves in a plate, with analytical solution and eigenvalues known exactly, and second, problems whose dispersion relation has (or not) been found analytically and whose numerical solution is an irrational number. Lamb waves belong to the second category, we know the solution analytically but the numerical values have a non-integral part which renders them a bad reference for numerical comparisons as it is unclear whether the root finder or the SCM is more accurate, indeed arguably SCM provides the reference value.

With problems of the first category, one can pursue accuracy and convergence studies by changing the number of grid points $N$ and comparing the results with the exact solution. This was done for $\mathrm{SH}$ modes in an isotropic plate, where the solutions are integers in the appropriate scale, and excellent agreement was found within the first half of the eigenvalues. Generally, the accuracy decreases as we move to higher eigenvalues, but there can be exceptions, that is, the third eigenvalue might be less accurate than the fourth. This is in complete agreement with the results shown in the literature [35] and [33]. The convergence towards the exact solution is also very good, with accuracy of up to 11 digits. As we increase $N$ the number of non-changing digits increases up to 11 which then, upon reaching a certain value of $N$, stay unchanged no matter how much we further increase $N$. 
In problems of the second category we do not have an exact reference to compare our numerical results with, an integer for example. Therefore, the most sensible way to proceed is to study the self-convergence by increasing $N$ and monitoring how the solution changes. This has been done for Lamb modes in a plate and the agreement with the PWRF methods was very high (as can be seen in Table II). We could also confirm that the number of unchanging digits increases up to 11 digits as we increase $N$ up to a certain value. From this $N$ value upwards these self-convergent digits stay the same. Again, and taking as reference the values given by PWRF routines, the rule by which the higher the eigenvalue is, the lower the accuracy, holds in general, although, not strictly.

We conclude that as a "rule of thumb" the accuracy of the first half of eigenvalues can be safely relied upon and that the results given by the SCM are self-convergent up to a very high number of digits. The accuracy of the results, when compared to the solution given by PWRF methods, is also very good. We note that the self-convergence of the results worsens as we handle more complicated problems, multi-layered cases for instance, where the number of self-convergent digits sometimes decreases to as low as 6; this notwithstanding, the accuracy is still far within the margins of acceptance.

\section{RESULTS FOR SINGLE AND MULTILAYER SYSTEMS}

Now we present further results obtained choosing examples that cover cases that were previously inaccessible.

\section{A. Single-Layer Systems}

To be as general as possible we look at a problem of circumferential propagation in an anisotropic cylinder. In equation (18) we show the usual form of the solution assumed for the case with circumferential propagation.

$$
\text { Circumferential Propagating Modes: } u_{j}=U_{j}(r) e^{i(k \theta-\omega t)} ; j=r, \theta, z
$$




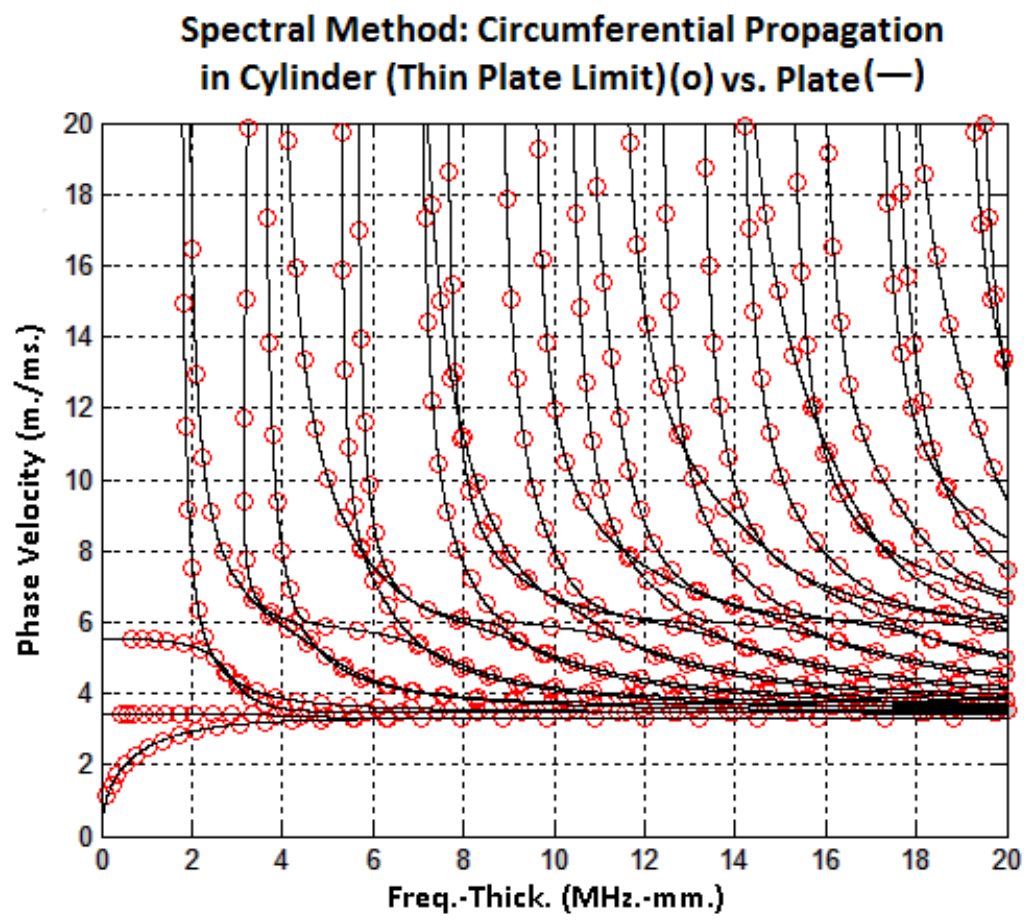

FIG. 9: Free Orthorhombic Cylinder with $\{Z\}$ crystal axis at 45 degrees with the $\{z\}$ spatial axis: Longitudinal circumferential propagating modes (solid lines) vs. Plate Lamb modes (circles).

Figure 9 displays the dispersion curves for circumferential propagation in a free cylinder for the thin plate limit cross-validated against its flat plate analogue. Both materials are Orthorhombic and, in the cylinder, the crystal axis $\{Z\}$ is at 45 degrees with the spatial axis $\{z\}$ which is aligned with the cylinder axis. Propagation is along the $\{\theta\}$ axis which is not parallel to the crystal $\{\Theta\}$ axis due to the rotation; from figure 9 both solutions are seen to coincide and agree very well. This general case is outside the scope of established PWRF routines, but poses no problem for the SCM.

\section{B. Multilayer Systems}

We now progress to multilayer systems. For clarity we present results where the number of layers is relatively low, but we have also extensively tested the scheme for many layered systems. The main change is that the time to plot the curves increases with the number of layers. Due to industrial interest in laminated composites and aggregates of plies made 
of different materials our first example is a system of three layers: isotropic-anisotropicisotropic.

We compare the results given by PWRF and the SCM in Figure 10 for the modes in this free plate three layer system. To be specific, we choose the anisotropic medium to be a GRE layer, a fibre composite material, and it is sandwiched between two steel layers. The axes configuration is the same in every layer: propagation along the principal axis $\{\mathrm{Z}\}$ and $\{Y\}$ axis perpendicular to the plane of the plate, so in this case the crystal axes and spatial axes are aligned.

This example is chosen to demonstrate how easily root searching methods such as the one used by the authors can miss pieces of the dispersion curves; the SCM finds the missing parts not entirely computed by PWRF. To be fair to PWRF, manual intervention can identify the missing parts by modifying the algorithm to perform a finer search, but we leave the lines broken to illustrate the point that SCM finds all solutions automatically without further intervention.

As noted earlier fluid layers are an important special case and so we close the section with an example of a multilayer system with a fluid layer placed between two solid layers. As explained in section II, lest we obtain spurious modes, the codes must be slightly modified when a perfect fluid layer is included between solid layers or at the core of the system (fluid-filled pipe for instance). The modification consists of using displacements for the spectral scheme of the solid layers and one single potential (longitudinal since we are dealing with inviscid fluids) for the spectral scheme of the fluid layer. Without loss of generality, this approach may be used satisfactorily for any isotropic or anisotropic solid layers. As an aside, the case of viscous fluids and exterior fluids surrounding the system require a different treatment not within the scope of this paper, but which will be the subject of future work.

Figure 11 displays the dispersion curves for the water layer sandwiched between two steel layers (with $5-2-5 \mathrm{~mm}$ thickness). The axes configuration is: propagation along the principal axis $\{\mathrm{Z}\}$ and $\{\mathrm{Y}\}$ axis perpendicular to the plane of the plate. The dispersion 


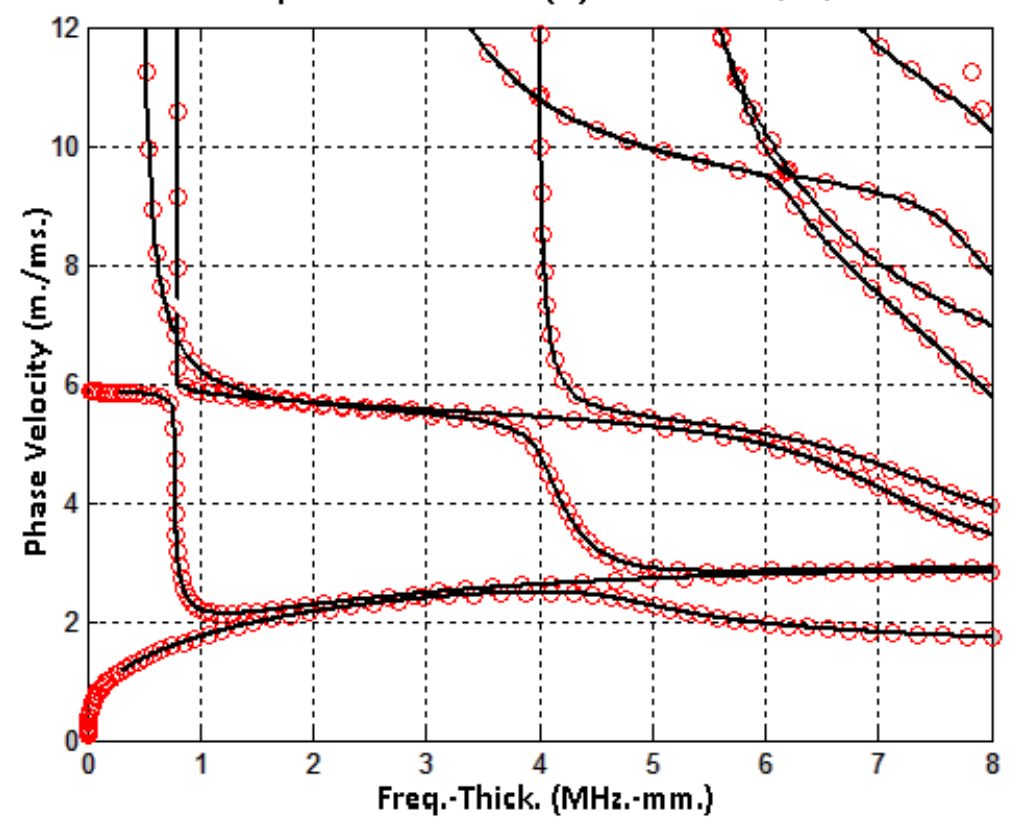

FIG. 10: Steel-GRE-Steel $(2-2-2 \mathrm{~mm}$ thick) Free Plate System: SCM (circles) vs. PWRF (solid lines). GRE is a fibre composite whose physical parameters are given in the appendix. The product frequency-thickness of the graph's $\{\mathrm{x}\}$ axis refers to the total thickness of the system.

curves display only Lamb modes, the SH modes have not been plotted since water, a perfect fluid, has no effect on them. Importantly, this figure also shows an example of a spurious mode found by the PWRF model. The horizontal line at $1.5 \mathrm{~m} / \mathrm{ms}$ is a spurious solution at the speed of bulk compression waves in the water. It is not found by the SCM.

Figure 12 displays the mode shapes of the first five modes in this three-layered system Steel-Water-Steel: this illustrates another advantage of the SCM, namely, we simultaneously obtain both the eigenvalues (modes) and eigenvectors (mode shapes). The mode shapes have the expected behaviour with the normal component of the displacement field continuous across the perfect fluid-solid interface whereas the tangential, or shear, component is discontinuous. It is notable that the mode shapes display no numerical noise or deviation despite the discontinuity.

Table III gives a typical numerical comparison between results obtained by SCM 
Lamb Modes Plate System Steel-Water-Steel:

Spectral Method (o) vs. PWRF (-)

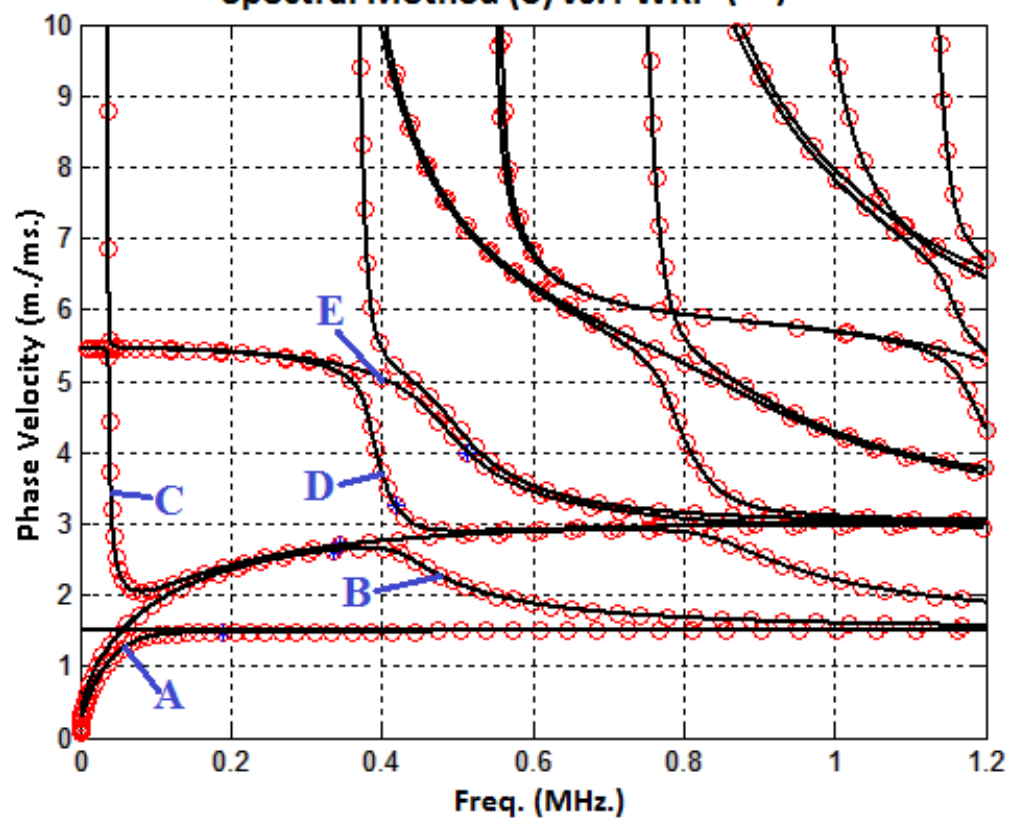

FIG. 11: Steel-Water-Steel (5-2-5 mm thick) Plate System: SCM (circles) vs. PWRF (solid lines). The axes' orientation is shown in Figure 1. The horizontal line at $1.5 \mathrm{~m} / \mathrm{ms}$ is a spurious solution of the partial waves method. It is not found by the SCM.

and PWRF, and we choose the example shown in Figure 11. The values are at points for the first 5 eigenmodes and are all well within the desired accuracy margin. Running the code for $N=110$ and computing the relative error between both sets of results shows the highest relative error between iterations lies below $10^{-3} \%$. Given the high accuracy of SCM it is not clear which of the methods should be the reference "gold-standard" computation, this minor offset between the codes is due to the higher complexity of the problem vis-a-vis the single layer case. None the less the results sufficiently confirm the good convergence and reliability of the solution.

We performed similar comparisons in cylindrical geometry again obtaining very good agreement between the SCM and PWRF. Moreover, we verified again that the SCM did not compute any spurious mode when a fluid layer is present (a water-filled pipe for instance), a well-known problem of the PWRF approach [17]. 
Spectral Method:

Lamb Modes' profiles for Steel-Water-Steel Plate System

Uy-displacemnt Profiles

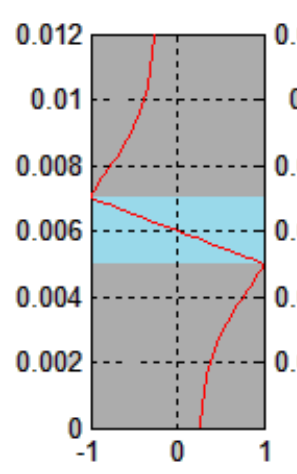

A

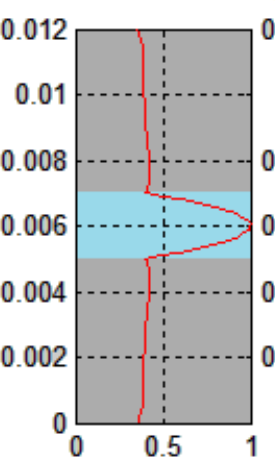

B

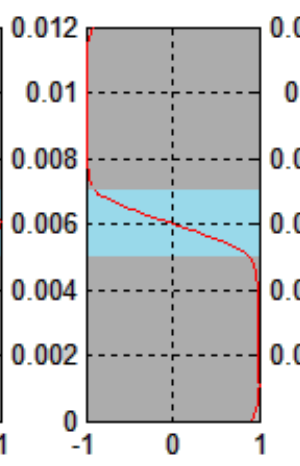

$\mathrm{C}$

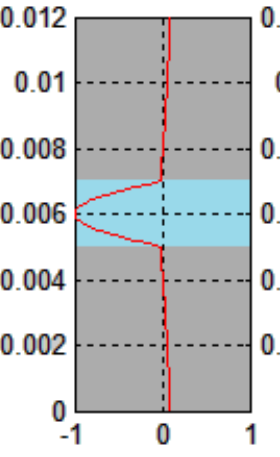

D

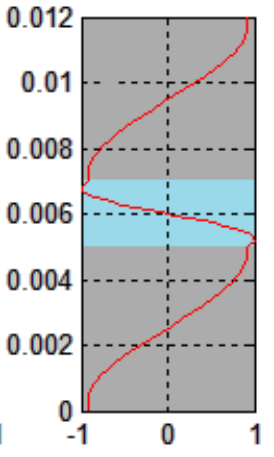

$\mathbf{E}$

Uz-displacement Profiles
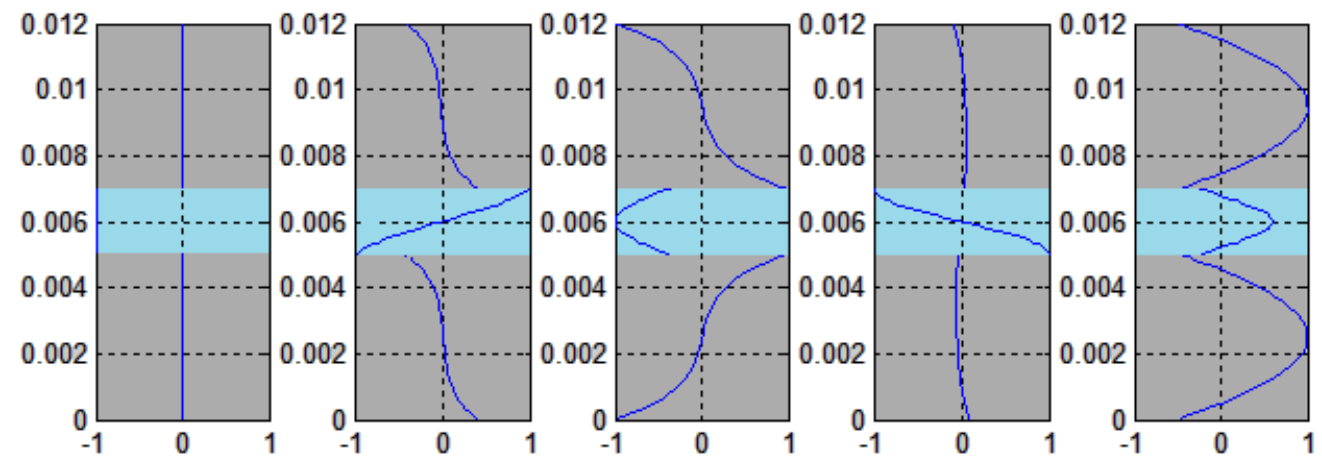

FIG. 12: Steel-Water-Steel (5-2-5 mm thick) Plate System: Displacement Profiles of the system in Figure 11. Vertical axes plot the thickness of the system. Horizontal axes are adimensional and plot the relative amplitude of the field normalized to the maximum value. Profiles obtained with the SCM.

\section{Multilayered Systems vs. Spring Interface}

To efficiently handle systems where an interface is modelled with finite stiffness, with very thin layers or has imperfect contact conditions, a spring interface is a sensible alternative. SCM codes for perfectly matched multi-layered systems require many more grid points when extreme cases exist, such as if the thicknesses of the constituent layers differ by several orders of magnitude ( 4 or more). We believe that this is caused by the difference, and subsequent numerical ill conditioning of the matrix, in the system's matrix entries due to the extreme difference in the thicknesses of the layers. For these cases the Spring Model 


\begin{tabular}{|c|c|c|c|c|}
\hline Mode & $\begin{array}{c}\text { Freq.-Thick. } \\
(\mathrm{MHz}-\mathrm{mm})\end{array}$ & $\begin{array}{c}\text { PWRF } \\
V_{p h}(\mathrm{~m} / \mathrm{ms})\end{array}$ & $\begin{array}{c}\text { S. } \mathbf{M} .(N=110) \\
V_{p h}(\mathrm{~m} / \mathrm{ms})\end{array}$ & $\begin{array}{c}\text { Relative Error } \\
10^{-3} \%\end{array}$ \\
\hline A & 0.476536 & 2.27840 & 2.27900 & 26.3340 \\
\hline B & 0.602921 & 2.88347 & 2.88350 & 1.0404 \\
\hline C & 0.609561 & 2.91533 & 2.91522 & 3.7731 \\
\hline D & 0.677135 & 3.23840 & 3.23840 & 0 \\
\hline E & 0.686913 & 3.28530 & 3.28510 & 6.0877 \\
\hline
\end{tabular}

TABLE III: Relative error between the results given by the SCM and PWRF for the case of Figure 11. The values correspond to the first 5 eigenvalues.

is preferable.

To cross-validate the Spring Model in the SCM we have run several examples in flat and cylindrical geometries and compared them to the results given by existing and validated PWRF routines.

Figure 13 features both, the solution given by the SCM and that given by a PWRF routine. For this example we used two steel $1 \mathrm{~mm}$ thick layers. The spring constants are $K_{t}=10^{12} \mathrm{~N} / \mathrm{m}^{3}$ and $K_{n}=10^{16} \mathrm{~N} / \mathrm{m}^{3}$, these are arbitrary values that have been chosen to be within the range where variation of the values influences the dispersion curves; that is to say, they are not approaching either of the limiting cases of a decoupled interface or a perfectly coupled interface.

As anticipated the SCM solution agrees very well with that provided by the PWRF routine. We have also investigated limiting cases with very high and very low spring constants and they reproduce the desired limiting cases of perfectly bonded layers and two unbonded layers respectively. Similarly, cross-verification for the cylindrical case in the thin plate limit shows perfect agreement with their corresponding flat analogues. 


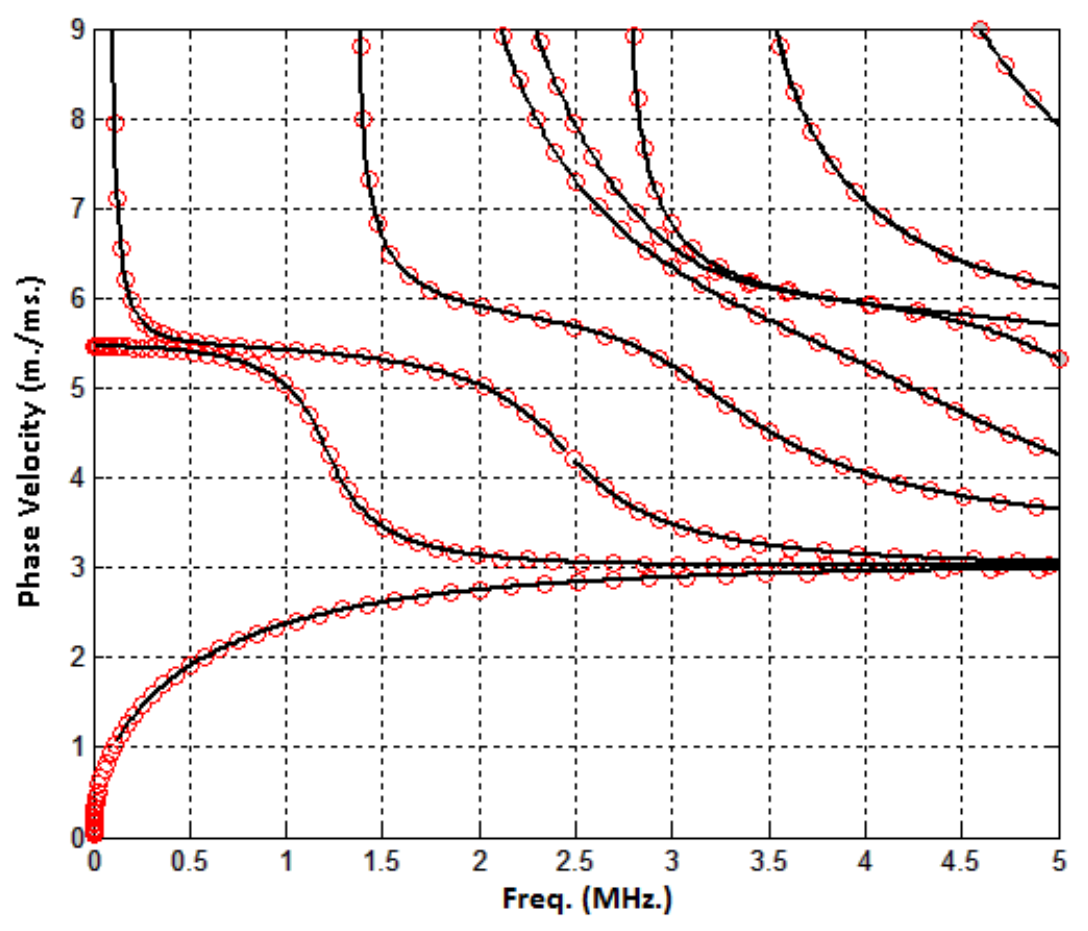

FIG. 13: Two 1mm thick Steel layers imperfectly bonded: SCM (circles) vs. PWRF (solid lines). The physical details of the spring interface can be found in the appendix. The spatial axes' configuration is shown in Figure 1. The plot features only the Lamb modes in the plate system.

\section{DISCUSSION AND CONCLUSIONS}

As we have demonstrated, the Spectral Method (SCM) provides a powerful alternative to partial-wave root-finding routines and the other methods described in our brief review in section I. We have comprehensively validated and cross-checked this by means of various comparisons with available literature and papers; we chose the most general and illustrative cases to present here. Additionally, several cases have also been validated with the established PWRF code.

The examples studied have highlighted the advantages of the SCM approach as compared to widely used PWRF routines: SCM overcomes the complexities and implementation difficulties of arbitrarily orientated axes and type of material encountered by PWRF for- 
mulations. It solves the most general cases of Triclinic materials and arbitrary orientations of the crystal axes, whether in cylindrical or in flat geometry. A key advantage is that SCM does not miss any modes, so we can always be sure that the modes which are computed are all the modes that exist for that given case and frequency/wavenumber range. This removes the tedious task of searching for candidate missing modes, and removes the uncertainty of missing modes encountered by the authors when using a general PWRF routine. In section IV-B we also verified that the SCM does not find any spurious modes in problems where PWRF routines do.

There is considerable industrial need for studying waves in all kinds of media and for multilayers, so we have further extended the SCM to more general multilayer systems than those only involving isotropic media [21] and [23], to include both anisotropic layers and fluid layers. The accuracy of the results is very high and they present excellent self-convergence even for more complicated cases with various layers. Very complicated cases do require some care and convergence should be checked for increasing values of $N$. This notwithstanding, the accuracy and self-convergence remain within, more than acceptable, margins even for the most complicated cases of multi-layered systems of anisotropic materials.

Without any special treatment SCM do not suffer from the "large- $f d$ " problem inherent to many PW based methods, such as the Transfer Matrix method. This has been confirmed by running a code twice for different $\mathrm{N}$ to test the convergence and accuracy of the first modes. We found that the modes are equally accurate for high values of the product " $f d "$ ". Accuracy and convergence of the results was verified for as high a value as $25 \mathrm{MHz}$-mm. in a $5 \mathrm{~mm}$. steel plate and we found no errors or inaccuracies (cf with the example for a titanium plate cited in [17] where the practical limit for the first two modes was set at 15 MHz.-mm.). Finally, on top of all the aforementioned advantages, the ease of coding makes SCM very convenient when compared to other available options for solving waveguide problems.

Future work will further develop this methodology to handle media with damping and for leaky systems embedded in fluid or solid media of infinite extension such that waves 
radiate from the waveguide. Also, implementation of the SCM in a working model with developed algorithms to link the point-by-point results into continuous lines for each mode.

\section{APPENDIX A: NUMERICAL DATA}

The physical and geometrical information used for the figures presented in the main text are given here; in certain cases we have used the same material in multiple figures. The number of grid points $N$ varies from one example to another, but it is always at least double the number of modes plotted in the figure. On a practical level we choose $N$ to achieve the shortest computation time, that is, if we are interested in the first 10 modes, running a code with $N=100$ is unnecessary; a value of $N$ between 25 and 30 has consistently been shown to be sufficient.

The parameters for the plate of Figure 3 are as follows (with the usual axes orientation):

$$
\rho=5300 \mathrm{~kg} / \mathrm{m}^{3} ; \quad h=5 \mathrm{~mm}
$$

$h$ stands for the thickness of the plate. The elastic stiffness matrix is given in GPa:

$$
\left(\begin{array}{cccccc}
23.9 & 10.4 & 5 & & & \\
& 24.7 & 5.2 & & & \\
& & 13.5 & & & \\
& & & 6.5 & & \\
& & & & 6.6 & \\
& & & & & 7.6
\end{array}\right)
$$

The parameters for the plate of Figure 4 are (note that the axis configuration is that used in [12], and detailed in the caption of the figure):

$$
\rho=8938.4 \mathrm{~kg} / \mathrm{m}^{3} ; \quad h=5 \mathrm{~mm} ; \quad V_{55}=\sqrt{c_{55} / \rho} \mathrm{m} / \mathrm{ms}
$$


The elastic stiffness matrix is given in GPa:

$$
\left(\begin{array}{rrrrrr}
182.1 & 119.1 & 111.8 & & & \\
& 167.7 & 126.2 & & & \\
& & 174.9 & & & \\
& & & 92.1 & & \\
& & & & 53.5 & \\
& & & & & 67.6
\end{array}\right)
$$

The parameters for the plate of Figure 5 are (with the usual axes orientation):

$$
\rho=8938.4 \mathrm{~kg} / \mathrm{m}^{3} ; \quad h=5 \mathrm{~mm} ; \quad V_{66}=\sqrt{c_{66} / \rho} \mathrm{m} / \mathrm{ms}
$$

The elastic stiffness matrix is given in GPa:

$$
\left(\begin{array}{cccccc}
207.87 & 109.06 & 93.41 & 16.57 & -16.15 & -23.18 \\
& 167.70 & 136.24 & -24.72 & 11.28 & 8.68 \\
& & 185.91 & 8.14 & 8.21 & 14.50 \\
& & & 100.23 & 14.50 & 5.84 \\
& & & & 35.11 & 16.57 \\
& & & & & 59.47
\end{array}\right)
$$

Parameters for the cylinder of figures 6 and 7 are (with the usual axes orientation):

$$
\rho=8938.4 \mathrm{~kg} / \mathrm{m}^{3} ; \quad h=5 \mathrm{~mm} ; \quad r_{i}=5 \cdot 10^{3} \mathrm{~mm}
$$

$r$ stands for the inner radius. The elastic stiffness matrix is the same as in (A6).

Parameters for Figure 8 are as follows (with the usual axes orientation):

$$
\rho=8938.4 \mathrm{~kg} / \mathrm{m}^{3} ; \quad h=100 \mathrm{~mm} ; \quad r_{i}=100 \mathrm{~mm}
$$

The elastic stiffness matrix is the one given in (A6).

Parameters for the cylinder of Figure 9 are as follows (with the $\{Z\}$ axis at 45 degrees with the spatial $\{z\}$ axis ):

$$
\rho=5300 \mathrm{~kg} / \mathrm{m}^{3} ; \quad h=1 \mathrm{~mm} ; \quad r_{i}=1 \cdot 10^{3} \mathrm{~mm}
$$


The elastic stiffness matrix is given in (A2).

Parameters for the three-plate system of Figure 10 are as follows (with the usual axes orientation): For the steel plates we have:

$$
\rho=7932 \mathrm{~kg} / \mathrm{m}^{3} ; \quad h=2 \mathrm{~mm} ; \quad \mu=84.29 \mathrm{GPa} ; \quad \lambda=113.16 \mathrm{GPa}
$$

For the GRE plate we have

$$
\rho=1605 \mathrm{~kg} / \mathrm{m}^{3} ; \quad h=2 \mathrm{~mm}
$$

The elastic stiffness matrix of the GRE layer is given in GPa:

$$
\left(\begin{array}{cccccc}
11.69 & 5.85 & 5.61 & & & \\
& 11.69 & 5.61 & & & \\
& & 130.19 & & & \\
& & & 3.7 & & \\
& & & & 3.7 & \\
& & & & & 2.92
\end{array}\right)
$$

Parameters for the system of Figure 11 are as follows (with the usual axes orientation). For the steel plate the thickness is $h=5 \mathrm{~mm}$ and the other physical parameters are those given in (A10). For the water layer we have:

$$
\rho=1000 \mathrm{~kg} / \mathrm{m}^{3} ; \quad h=2 \mathrm{~mm} ; \quad \mu=0 \mathrm{GPa} ; \quad \lambda=2.25 \mathrm{GPa}
$$

The stiffness matrix in rectangular coordinates must be rearranged in a similar fashion to [16] when dealing with problems in cylindrical coordinates.

The system of Figure 13 is composed of two imperfectly coupled steel layers. The two steel layers are each $1 \mathrm{~mm}$ thick, with properties given in (A10). The spring constants for the interface are $K_{t}=10^{12} \mathrm{~N} / \mathrm{m}^{3}$ and $K_{n}=10^{16} \mathrm{~N} / \mathrm{m}^{3}$. 


\section{REFERENCES}

1 B. Pavlakovic, M. Lowe, D. Alleyne, and P. Cawley, "DISPERSE: A general purpose program for creating dispersion curves," Review of Progress in Quantitative Non-destructive Evaluation 16, 185 (1987).

2 B. Pavlakovic and M. Lowe, DISPERSE User's Manual, Version 2.0.20B (2013), pp. 1-209.

3 K. F. Graff, Rayleigh and Lamb Waves (Dover, New York, 1991), pp. 1-649.

4 B. A. Auld, Acoustic Fields and Waves in Solids, 2nd Ed. (Krieger Publishing Company, Florida, 1990), pp. 1-878.

5 J. L. Rose, Ultrasonic Waves in Solid Media (Cambridge University Press, Cambridge, 1999), pp. $1-476$.

6 J. D. Achenbach, Wave propagation in elastic solids (North-Holland, Amsterdam, 1973), pp. $1-440$.

7 R. D. Mindlin, "Waves and vibrations in isotropic, elastic plates," Structural Mechanics. (Eds. J.N. Goodier and N. Hoff) 199-323 (1960).

8 Y.-H. Pao, "The dispersion of Flexural waves in an elastic circular cylinder, Part II," Journal of Applied Mechanics 29, 61-64 (1962).

9 L. P. Solie and B. A. Auld, "Elastic waves in free anisotropic plates," Journal of the Acoustical Society of America 54 (1), 50-65 (1973).

10 A. H. Nayfeh and D. E. Chimenti, "Free Wave Propagation in Plates of General Anisotropic Media," in D. Thompson and D. Chimenti, editors, Review of Progress in Quantitative Nondestructive Evaluation (Plenum Press, New York, 1989), 181.

11 A. H. Nayfeh and D. E. Chimenti, "Free Wave Propagation in Plates of General Anisotropic Media," Journal of Applied Mechanics 56, 881-886 (1989).

12 Y. Li and R. B. Thompson, "Influence of anisotropy on the dispersion characteristics of guided ultrasonic plate modes," Journal of the Acoustical Society of America 87 (5), 1911-1931 (1990).

13 M. Onoe, H. D. McNiven, and R. D. Mindlin, "Dispersion of axially symmetric waves in elastic rods," Journal of Applied Mechanics 29, 61-64 (1962).

14 R. D. Mindlin and Y.-H. Pao, "Dispersion of Flexural waves in elastic circular cylinder," Journal 
of Applied Mechanics 27, 513-520 (1960).

15 J. Zemanek, "An Experimental and Theoretical Investigation of Elastic Wave Propagation in a Cylinder," Journal of the Acoustical Society of America 51, 265-283 (1972).

16 S. Towfighi, T. Kundu, and M. Ehsani, "Elastic wave propagation in circumferential direction in anisotropic cylindrical curved plates," Journal of Applied Mechanics 69, 283-291 (2002).

17 M. Lowe, "Matrix Techniques for modeling ultrasonic waves in multilayered media," IEEE Trans. Ultrason. Ferroelectr. Freq. Control 42, 525-542 (1995).

18 A. H. Nayfeh, "The propagation of horizontally polarized shear waves in multilayered anisotropic media," Journal of the Acoustical Society of America 86 (5), 2007-2012 (1989).

19 A. H. Nayfeh, "The general problem of elastic wave propagation in multilayered anisotropic media," Journal of the Acoustical Society of America 89 (4), 1521-1531 (1990).

20 R. Vasudeva, G. Sudheer, and A. Vema, "Dispersion of circumferential waves in cylindrically anisotropic layered pipes in plane strain," Journal of the Acoustical Society of America 123 (6), 4147-4151 (2008).

21 F. Karpfinger, B. Gurevich, and A. Bakulin, "Modeling of wave dispersion along cylindrical structures using the spectral method," Journal of the Acoustical Society of America 124 (2), 859-865 (2008).

22 A. Adamou and R. Craster, "Spectral methods for modelling guided waves in elastic media," Journal of the Acoustical Society of America 116 (3), 1524-1535 (2004).

23 B. Yu, S. Yang, C. Gan, and H. Lei, "A New Procedure for Exploring the Dispersion Characteristics of Longitudinal Guided Waves in a Multilayered Tube with Weak Interface," Journal of Nondestructive Evaluation 32 (3), 263-276 (2013).

24 D. Levesque and L. Piche, "A robust transfer matrix formulation for the ultrasonic response of multi-layered absorbing media," J. Acoust. Soc. am. 92, 452-467 (1992).

25 S. Rokhlin and Y. Wang, "Stable recursive algorithm for elastic wave propagation in layered anisotropic media: stiffness matrix method," Journal of the Acoustical Society of America 112 (3), 822-834 (2002).

26 B. Hosten and M. Castaings, "Surface impedance matrices to model the propagation in multilayered media," Ultrasonics 41, 501-507 (2003).

27 L. Gavric, "Computation of propagative waves in free rail using a finite element technique," Journal of Sound Vibration 185, 531543 (1995). 
M. Castaings and M. Lowe, "Finite Element model for waves guided along solid systems of arbitrary section coupled to infinite solid media," Journal of the Acoustical Society of America 123 (2), 696-708 (2008).

29 M. Castaings, C. Bacon, M. Lowe, and Z. Fan, "Torsional waves propagation along a waveguide of arbitrary cross section immersed in a perfect fluid," Journal of the Acoustical Society of America 124 (4), 2002-2010 (2008).

30 M. Lowe and Z. Fan, "Elastic waves guided by a welded joint in a plate," Proceedings of the Royal Society A 2009465124 (4), 2053-2068 (2009).

31 I. Bartoli, A. Marzani, F. Lanza di Scalea, and E. Viola, "Modeling wave propagation in damped waveguides of arbitrary cross-section," Journal of Sound and Vibration 295, 685-707 (2006).

32 A. Marzani, E. Viola, I. Bartoli, F. Lanza di Scalea, and P. Rizzo, "A semi-analytical finite element formulation for modeling stress wave propagation in axisymmetric damped waveguides," Journal of Sound and Vibration 318, 488-505 (2008).

33 L. Trefethen, Spectral Methods in MATLAB (SIAM, Philadelphia, 2000), pp. 1-181.

34 J. Weideman and S. Reddy, "A MATLAB differentiation matrix suite," ACM Transactions on Mathematical Software 26 (4), 465-519 (2000).

35 J. P. Boyd, Chebyshev and Fourier Spectral Methods (Dover, New York, 2001), pp. 1-688.

36 B. Fornberg, A Practical Guide to Pseudoespectral Methods (1996), pp. 1-231.

37 F. Karpfinger, B. Gurevich, and A. Bakulin, "Modeling of axisymmetric wave modes in a poroelastic cylinder using the spectral method," Journal of the Acoustical Society of America 124 (4), EL230-EL235 (2008).

38 F. Karpfinger, H. Valero, B. Gurevich, A. Bakulin, and B. Sinha, "Spectral-method algorithm for modeling dispersion of acoustic modes in elastic cylindrical structures," Geophysics $\mathbf{7 5}$ (3), H19-H27 (2010).

39 T. Zharnikov, D. Syresin, and C.-J. Hsu, "Application of the spectral method for computation of spectrum of anisotropic waveguides," in Proceedings on Meetings on Acoustics (Acoustical Society of America, 2013).

40 S. Rokhlin and Y. Wang, "Analysis of boundary conditions for elastic wave interaction with an interface between two solids," Journal of the Acoustical Society of America 89 (2), 503-515 (1991).

41 E. Dieulesaint and D. Royer, Elastic Waves in Solids (Springer Verlag, Berlin Heidelberg, 1996), 
pp. 1-392.

42 J. Kaplunov, L. Y. Kossovich, and E. Nolde, Dynamics of thin walled elastic bodies (Elsevier, New York, 1997), pp. 1-240.

43 C. Wang, J. Reddy, and K. Lee, Shear deformable beams and plates. Relationships with classical solutions. (Elsevier, New York, 2000), pp. 1-312. 